ALEA, Lat. Am. J. Probab. Math. Stat. 17, 23-49 (2020)

DOI: $10.30757 /$ ALEA.v17-02

\title{
Dynamical coupling between Ising and FK percolation
}

\section{Raphaël Cerf and Sana Louhichi}

DMA, Ecole Normale Supérieure, CNRS, PSL Research University, 75005 Paris.

Laboratoire de Mathématiques d'Orsay,

Université Paris-Sud, CNRS, Université Paris-Saclay, 91405 Orsay.

E-mail address: raphael.cerf@ens.fr

URL: https://www.math.u-psud.fr/ cerf/

Laboratoire Jean Kuntzmann.

Bâtiment IMAG, 700 avenue centrale

38400 Saint Martin d'Hères, France.

E-mail address: sana.louhichi@univ-grenoble-alpes.fr

URL: http://www-ljk.imag.fr/membres/Sana.Louhichi/

\begin{abstract}
We investigate the problem of constructing a dynamics on edge-spin configurations which realizes a coupling between a Glauber dynamics of the Ising model and a dynamical evolution of the percolation configurations. We dreamed of constructing a Markov process on edge-spin configurations which is reversible with respect to the Ising-FK coupling measure, and such that the marginal on the spins is a Glauber dynamics, while the marginal on the edges is a Markovian evolution. We present two local dynamics, one which fulfills only the first condition and one which fulfills the first two conditions. We show next that our dream process is not feasible in general. We present a third dynamics, which is non local and fulfills the first and the third conditions. We finally present a localized version of this third dynamics, which can be seen as a contraction of the first dynamics.
\end{abstract}

\section{Introduction}

The Ising model, invented almost a century ago, is one of the most studied models of statistical mechanics. A fundamental tool to analyze the Ising model is the random cluster model, or FK model, invented by Fortuin and Kasteleyn around 1969 (see the reference book Grimmett, 2006). Results on this dependent percolation model can be transferred towards the Ising model via a coupling construction

Received by the editors May 28th, 2019; accepted November 11th, 2019.

2010 Mathematics Subject Classification. 60J75, 60J27, 60K35, 82B20, 82B43.

Key words and phrases. Ising model, FK percolation, Random-cluster process, Glauber dynamics, Coupling measure, Reversibility. 
due to Edwards and Sokal (1988). This machinery works extremely well, but so far, it has essentially only been employed to study the systems at equilibrium. Yet another rich facet of the Ising model is the dynamics. There exist several microscopic dynamics on spin configurations, which give rise to the so-called stochastic Ising model, and whose equilibrium are described by the Ising Gibbs measure: the Metropolis dynamics, the heat-bath dynamics, the Kawasaki dynamics, to name a few of them. On one hand, these dynamics provide a basic model to study fundamental questions on dynamics, for instance to model the metastability phenomenon (Olivieri and Vares, 2005). On the other hand, there is a hope that the understanding of the dynamics will shed light on the equilibrium measure.

Many percolation models possess also a natural dynamics. For the Bernoulli percolation model, it consists of independently updating the edges, and it leads to beautiful difficult problems (Häggström, 1998; Steif, 2009). For the FK model, the construction of a dynamics is more subtle and it typically involves non-local computations (Grimmett, 1995).

One naturally wonders whether the stochastic Ising model and dynamical percolation could help to understand each other: Is it possible to construct a valuable dynamical coupling on edge-spins configurations? Such a coupling, if successful, would allow to transfer some techniques from percolation theory to spin dynamics. The idea would be to recast spin dynamics as dynamics on the edges, as it was the case for the edge-spin coupling measure. As far as we know, the question of constructing such a dynamical coupling has not been addressed before. Coupling is a very powerful tool and a general technique in the theory of interacting particle systems. We refer, for instance, to Chapter II in Liggett (2005) for its use and its application to Markov chains.

Our note is an investigation into the possibility of building a coupling for the dynamics on Ising and percolation models, which could help to understand the dynamics of the Ising model. We focus here on the case of a Glauber type dynamics, that is a local dynamics which modifies at most one spin at a time. The precise definition of such dynamics, together with several examples, are given in section 3.

We dreamed of building a coupling dynamics on edge-spins configurations such that:

- The marginal on the spins is a Glauber dynamics.

- The marginal on the edges is a simple Markovian evolution.

- The coupling dynamics on edge-spins configurations is reversible with respect to the coupling measure between the Ising and the FK models.

Notice that if we drop the first constraint, then we could simply consider a time continuous Markov process on the edge-spins configurations which, after an exponential time of parameter one, jumps on a new independent edge-spins configuration, drawn according to the Ising-FK coupling measure. However the first constraint is essential for us, indeed our hope is to build a dynamical coupling which would help to study a Glauber dynamics of the Ising model.

The definition of the Ising-FK coupling measure is recalled in section 2. The stochastic Ising model is defined in section 3. The FK dynamics is defined in section 4 . The above conditions are precisely stated in section 5 . We manage to build couplings which satisfy the third condition. The most basic coupling is a dynamics which changes at most one object at each step, i.e., either one spin or one edge is modified at a time. We prove that, for this type of coupling, the first condition can 
never be satisfied. We build such a coupling dynamics in section 6 . Another natural dynamics consists in changing simultaneously the spin at one vertex, together with the edges incident to this vertex. Such a dynamics is presented in section 7 . The good news is that the marginal of this dynamics on the spins is a Glauber dynamics. However the marginal of this type of dynamics on the edges is not Markovian. In section 8 , we show that our dream process is not feasible in general. We present a third dynamics in section 9, which is non local and fulfills the first and the third conditions. We finally present in section 10 a localized version of this third dynamics, which can be seen as a contraction of the first dynamics.

\section{Ising-FK coupling measure}

In this section, we recall some classical notation and we define the Ising-FK coupling measure IP (IP for Ising-Percolation). We consider a finite graph $(V, E)$, where $V$ is the set of the vertices and $E$ the set of the edges. The edges are unoriented, and $E$ is a subset of the set of pairs of points of $V$. Throughout the paper, we suppose that the set of edges $E$ is not empty. An edge configuration $\eta$ is an element of $\{0,1\}^{E}$ (where 0 stands for closed and 1 for open). A spin configuration $\sigma$ is an element of $\{-1,1\}^{V}$. An edge $e$ with endvertices $x$ and $y$ is written as $e=\langle x, y\rangle$ or $e=\langle y, x\rangle$. We define

$$
\forall \sigma \in\{-1,1\}^{V} \quad \forall e=\langle x, y\rangle \in E \quad \delta_{\sigma}(e)=\mathbb{I}_{\sigma(x)=\sigma(y)} .
$$

An edge configuration $\eta$ and a spin configuration $\sigma$ are said to be compatible if we have

$$
\forall e \in E \quad \eta(e) \leq \delta_{\sigma}(e),
$$

i.e., if the endvertices of any open edge in $\eta$ have the same spins in $\sigma$. We denote by $\mathcal{C}$ the set of the pairs of compatible configurations:

$$
\mathcal{C}=\left\{(\eta, \sigma) \in\{0,1\}^{E} \times\{-1,1\}^{V}: \eta(e) \leq \delta_{\sigma}(e) \text { for any } e \in E\right\} .
$$

Let $p$ be a fixed number in $[0,1]$. The Ising-Percolation measure $I P$ is the probability measure on the product space $\{0,1\}^{E} \times\{-1,+1\}^{V}$ defined as follows. For any $(\eta, \sigma)$ in $\{0,1\}^{E} \times\{-1,+1\}^{V}$, we set

$$
I P(\eta, \sigma)=\frac{1}{Z} \prod_{e \in E}\left(p \mathbb{I}_{\eta(e)=1} \delta_{\sigma}(e)+(1-p) \mathbb{I}_{\eta(e)=0}\right),
$$

where $Z$ is the partition function, i.e., the normalising constant that makes $I P$ a probability measure on $\{0,1\}^{E} \times\{-1,+1\}^{V}$, given by

$$
Z=\sum_{(\eta, \sigma) \in\{0,1\}^{E} \times\{-1,+1\}^{V}} \prod_{e \in E}\left(p \mathbb{I}_{\eta(e)=1} \delta_{\sigma}(e)+(1-p) \mathbb{I}_{\eta(e)=0}\right) .
$$

The Ising-Percolation measure can also be written as

$$
I P(\eta, \sigma)=\frac{1}{Z} p^{|\{e \in E: \eta(e)=1\}|}(1-p)^{|\{e \in E: \eta(e)=0\}|} \mathbb{I}_{(\eta, \sigma) \in \mathcal{C}},
$$

where the absolute value of a set denotes its cardinality. In fact, the measure $I P$ is the Bernoulli product measure on edge-spin configurations with parameters $(p, 1 / 2)$ conditioned to the set $\mathcal{C}$ of the compatible configurations. It is well-known that the probability measure $I P$ is a coupling of the Ising measure $\mu_{\beta}$ defined on $\{-1,+1\}^{V}$, 
together with the random-cluster measure $\phi_{p, 2}$ defined on $\{0,1\}^{E}$, with the relation $p=1-e^{-\beta}$. In fact, the first marginal measure $\phi_{p, 2}$ on $\{0,1\}^{E}$ is given by

$$
\begin{aligned}
\forall \eta \in\{0,1\}^{E} \quad \phi_{p, 2}(\eta)= & \sum_{\sigma \in\{-1,+1\}^{V}} I P(\eta, \sigma) \\
& =\frac{1}{Z_{R C}}\left(\prod_{e \in E} p^{\eta(e)}(1-p)^{1-\eta(e)}\right) 2^{k(\eta) .}
\end{aligned}
$$

Here $k(\eta)$ denotes the number of connected components (or open clusters) of the graph having for vertices $V$ and edges the open edges in the configuration $\eta$. Note that isolated vertices, which are vertices incident to no open edge, are clusters of size one and they are included in the count of $k(\eta)$ (recall that an open cluster in $\eta$ is a set of vertices which are connected by open edges in $\eta$, recall also that an edge $e$ is said to be open in $\eta$ if $\eta(e)=1$ and closed if $\eta(e)=0)$. Naturally, $Z_{R C}$ is the partition function that makes $\phi_{p, 2}$ a probability measure on $\{0,1\}^{E}$. The second marginal measure is given by

$$
\forall \sigma \in\{-1,+1\}^{V} \quad \sum_{\eta \in\{0,1\}^{E}} I P(\eta, \sigma)=\frac{1}{Z}(1-p)^{\left|\left\{e \in E: \delta_{\sigma}(e)=0\right\}\right|} .
$$

Notice that

$$
\left|\left\{e \in E: \delta_{\sigma}(e)=0\right\}\right|=\sum_{e \in E}\left(1-\delta_{\sigma}(e)\right)=|E|-\sum_{e \in E} \delta_{\sigma}(e) .
$$

Let $\beta>0$ be such that $p=1-e^{-\beta}$. Rewriting the formula (2.3) with these notations, we see that the second marginal is the measure $\mu_{\beta}$ on $\{-1,+1\}^{V}$ given by

$$
\forall \sigma \in\{-1,+1\}^{V} \quad \mu_{\beta}(\sigma)=\frac{1}{Z_{I}} \exp \left(\beta \sum_{e \in E} \delta_{\sigma}(e)\right),
$$

where the partition function $Z_{I}$ is

$$
Z_{I}=Z \exp (\beta|E|) .
$$

Let us rewrite the Hamiltonian in a more classical way. For an edge $e$ with endpoints $x, y$, we have

$$
\delta_{\sigma}(e)=\frac{1}{2}(1+\sigma(x) \sigma(y)) .
$$

Summing over $e \in E$, we get

$$
\sum_{e \in E} \delta_{\sigma}(e)=\sum_{\{x, y\} \in E} \frac{1}{2}(1+\sigma(x) \sigma(y))=\frac{1}{2}|E|+\frac{1}{2} \sum_{\{x, y\} \in E} \sigma(x) \sigma(y) .
$$

The standard Hamiltonian of the Ising model is defined as

$$
\forall \sigma \in\{-1,+1\}^{V} \quad H(\sigma)=-\frac{1}{2} \sum_{\{x, y\} \in E} \sigma(x) \sigma(y) .
$$

In the end, we have

$$
\forall \sigma \in\{-1,+1\}^{V} \quad \mu_{\beta}(\sigma)=\frac{1}{Z_{\beta}} \exp (-\beta H(\sigma)),
$$


where the partition function $Z_{\beta}$ is given by

$$
Z_{\beta}=\sum_{\sigma \in\{-1,+1\}^{V}} \exp (-\beta H(\sigma))=Z_{I} \exp \left(-\frac{1}{2} \beta|E|\right) .
$$

We refer to the book Grimmett (2006) and the references therein for more details about the coupling measure $I P$ between random-cluster and Ising measures, its history and usefulness. We refer to the paper Schonmann (1994) for a nice presentation of the Gibbs measure of the Ising model and the associated dynamics.

\section{The stochastic Ising model}

In this section, we define Glauber type dynamics for the Ising model. Each of these dynamics defines a Markov process $\left(\sigma_{t}\right)_{t \geq 0}$ on the spin configurations which is called a stochastic Ising model. A Markov process $\left(\sigma_{t}\right)_{t \geq 0}$ on the spin configurations is classically defined through its infinitesimal generator $L$ (see Liggett, 2005). This generator $L$ acts on functions $f$ of the spin configuration and it is of the following form. For any function $f:\{-1,+1\}^{V} \rightarrow \mathbb{R}$, we have

$$
\forall \sigma \in\{-1,+1\}^{V} \quad L f(\sigma)=\sum_{\sigma^{\prime} \in\{-1,+1\}^{V}} c\left(\sigma, \sigma^{\prime}\right)\left(f\left(\sigma^{\prime}\right)-f(\sigma)\right) .
$$

The quantity $c\left(\sigma, \sigma^{\prime}\right)$ is the rate at which the configuration $\sigma$ is transformed into $\sigma^{\prime}$ when the system is in the state $\sigma$. We are usually interested in local dynamics which modify at most one spin at a time. We call this type of dynamics Glauber type dynamics. For a configuration $\sigma$ and a vertex $x$, we denote by $\sigma^{x}$ the configuration obtained from $\sigma$ by flipping the spin at the site $x$. So we require that $c\left(\sigma, \sigma^{\prime}\right)$ vanishes when $\sigma$ and $\sigma^{\prime}$ differ in more than one spin and the quantity $c\left(\sigma, \sigma^{x}\right)$, sometimes denoted by $c(x, \sigma)$, is the rate at which the spin at the site $x$ flips when the system is in the state $\sigma$. The transition rates $c(\cdot, \cdot)$ are defined on $\{-1,1\}^{V} \times$ $\{-1,1\}^{V}$ and have thus the following properties: for any $\sigma \in\{-1,+1\}^{V}$ and $x \in V$,

$$
\begin{aligned}
& c\left(\sigma, \sigma^{\prime}\right)=0 \text { if }\left|\left\{x \in V, \sigma(x) \neq \sigma^{\prime}(x)\right\}\right| \geq 2, \\
& c\left(\sigma, \sigma^{x}\right)>0 \text { for any } x \in V, \\
& c(\sigma, \sigma)=-\sum_{y \in V} c\left(\sigma, \sigma^{y}\right) .
\end{aligned}
$$

We say that the transition rates $c(\cdot, \cdot)$ satisfy the detailed balance condition with respect to the Ising measure $\mu_{\beta}$ if

$$
\forall \sigma \in\{-1,1\}^{V} \quad \forall x \in V \quad \mu_{\beta}(\sigma) c\left(\sigma, \sigma^{x}\right)=\mu_{\beta}\left(\sigma^{x}\right) c\left(\sigma^{x}, \sigma\right) .
$$

When this detailed balance condition holds, the associated dynamics is reversible with respect to the Ising measure $\mu_{\beta}$. Several rates satisfy the conditions (3.1) and the detailed balance condition (3.2). These dynamics and their fundamental properties are presented in the paper Schonmann (1994). In the same paper appears the graphical construction of these dynamics, which provides a natural and intuitive picture of the associated Markov processes. Let us present some classical choices. Recalling the definition of the Ising Hamiltonian (2.6), we define further, for $\sigma \in$ $\{-1,+1\}^{V}$ and $x \in V$,

$$
\Delta_{x} H(\sigma)=H\left(\sigma^{x}\right)-H(\sigma)
$$


For dynamics which modify at most one spin at a time, we set

$$
c(x, \sigma)=c\left(\sigma, \sigma^{x}\right) .
$$

Here are some possible choices for the rates:

The Metropolis dynamics:

$$
c(x, \sigma)=\exp \left(-\beta \max \left(\Delta_{x} H(\sigma), 0\right)\right) .
$$

The Heat bath dynamics:

$$
c(x, \sigma)=\frac{1}{\left.1+\exp \left(\beta \max \left(\Delta_{x} H(\sigma), 0\right)\right)\right)} .
$$

\section{Unnamed dynamics:}

$$
c(x, \sigma)=\exp \left(-\frac{\beta}{2} \Delta_{x} H(\sigma)\right) .
$$

These three choices satisfy the detailed balance condition (3.2) and correspond to one spin flip dynamics. We call them Glauber type dynamics. Let us try to express the rates with the help of the functions $\delta_{\sigma}$ on the edges. Using the identity (2.5), we have, for any $x \in V$,

$$
\Delta_{x} H(\sigma)=\sum_{y \in V:\{x, y\} \in E} \sigma(x) \sigma(y)=\sum_{e \in E_{x}}\left(2 \delta_{\sigma}(e)-1\right),
$$

where the set $E_{x}$ is the set of the edges $e \in E$ having $x$ as endvertex, i.e.,

$$
E_{x}=\{e=\langle x, y\rangle \in E: y \in V\} .
$$

For the unnamed dynamics, we obtain

$$
c(x, \sigma)=\exp \left(-\beta \sum_{e \in E_{x}} \delta_{\sigma}(e)+\frac{\beta}{2}\left|E_{x}\right|\right) .
$$

On the lattice $\mathbb{Z}^{d}$, we have $\left|E_{x}\right|=2 d$ for any $x \in \mathbb{Z}^{d}$. Up to a constant multiplicative factor, the previous rates are equal to

$$
c(x, \sigma)=\exp \left(-\beta \sum_{e \in E_{x}} \delta_{\sigma}(e)\right) .
$$

We will mostly use this choice when trying to build a dynamical coupling between the Ising model and the FK model.

\section{FK dynamics}

In this section, we define the counterpart of a Glauber type dynamics for the FK model. We are interested in dynamics which modify at most one edge at a time. We build a Markov process on percolation configurations, which is defined through its infinitesimal generator $L$ (see Liggett, 2005). This generator $L$ acts on functions $g$ of the percolation configuration and it is of the following form. For any function $g:\{0,1\}^{E} \rightarrow \mathbb{R}$, we have

$$
\forall \eta \in\{0,1\}^{E} \quad L g(\eta)=\sum_{e \in E} c\left(\eta, \eta^{e}\right)\left(g\left(\eta^{e}\right)-g(\eta)\right),
$$

where $\eta^{e}$ is the configuration obtained from $\eta$ by changing the state of the edge $e$ and the quantity $c\left(\eta, \eta^{e}\right)$ is the rate at which the edge $e$ changes its state when the 
system is in the state $\eta$. The transition rates $c(\cdot, \cdot)$ are defined on $\{0,1\}^{E} \times\{0,1\}^{E}$ and have the following properties: for any $\eta \in\{0,1\}^{E}$ and $e \in E$,

$$
\begin{aligned}
& c\left(\eta, \eta^{\prime}\right)=0 \text { if }\left|\left\{e \in E, \eta(e) \neq \eta^{\prime}(e)\right\}\right| \geq 2, \\
& c\left(\eta, \eta^{e}\right)>0 \text { for any } e \in E, \\
& c(\eta, \eta)=-\sum_{e \in E} c\left(\eta, \eta^{e}\right) .
\end{aligned}
$$

We say that the transition rates $c(\cdot, \cdot)$ satisfy the detailed balance condition with respect to the FK measure $\phi_{p, 2}$ if

$$
\forall \eta \in\{0,1\}^{E} \quad \forall e \in E \quad \phi_{p, 2}(\eta) c\left(\eta, \eta^{e}\right)=\phi_{p, 2}\left(\eta^{e}\right) c\left(\eta^{e}, \eta\right) .
$$

When this detailed balance condition holds, the associated dynamics is reversible with respect to the FK measure $\phi_{p, 2}$. Several rates satisfy the conditions (4.1) and the detailed balance condition (4.2). A natural choice is the following. For an edge $e=\langle x, y\rangle \in E$ and a configuration $\eta \in\{0,1\}^{E}$, we set $\gamma_{\eta}(e)=1$ if the endpoints $x, y$ of $e$ are connected by a path of open edges in $\eta$ which does not use the edge $e$ itself, and we set $\gamma_{\eta}(e)=0$ otherwise. We define then

$$
c(e, \eta):=c\left(\eta, \eta^{e}\right)= \begin{cases}1-p & \text { if } \eta(e)=1, \\ p & \text { if } \eta(e)=0 \text { and } \gamma_{\eta}(e)=1, \\ p / 2 & \text { if } \eta(e)=0 \text { and } \gamma_{\eta}(e)=0 .\end{cases}
$$

One can check that these rates satisfy (4.1) and (4.2).

\section{Our dream process}

The main purpose of this paper is to construct a Markov process on the space $\{0,1\}^{E} \times\{-1,+1\}^{V}$ whose marginal on the spins is a Glauber dynamics and which is reversible with respect to the coupling measure IP. Suppose that $\left(\eta_{t}, \sigma_{t}\right)_{t \geq 0}$ is such a Markov process and let $q\left((\eta, \sigma),\left(\eta^{\prime}, \sigma^{\prime}\right)\right)$ be its transition rates. These transition rates satisfy the following usual conditions:

$$
(\eta, \sigma) \neq\left(\eta^{\prime}, \sigma^{\prime}\right) \quad \Longrightarrow \quad q\left((\eta, \sigma),\left(\eta^{\prime}, \sigma^{\prime}\right)\right) \geq 0,
$$

and for any $(\eta, \sigma) \in\{0,1\}^{E} \times\{-1,+1\}^{V}$,

$$
\sum_{\left(\eta^{\prime}, \sigma^{\prime}\right) \in\{0,1\}^{E} \times\{-1,+1\}^{V}} q\left((\eta, \sigma),\left(\eta^{\prime}, \sigma^{\prime}\right)\right)=0 .
$$

The reversibility property with respect to the coupling measure $I P$ is equivalent to the following detailed balance equation:

$$
\begin{aligned}
& \forall(\eta, \sigma),\left(\eta^{\prime}, \sigma^{\prime}\right) \in\{0,1\}^{E} \times\{-1,1\}^{V} \\
& \quad I P(\eta, \sigma) q\left((\eta, \sigma),\left(\eta^{\prime}, \sigma^{\prime}\right)\right)=I P\left(\eta^{\prime}, \sigma^{\prime}\right) q\left(\left(\eta^{\prime}, \sigma^{\prime}\right),(\eta, \sigma)\right) .
\end{aligned}
$$

In general, the marginals of a Markov process are not themselves Markov processes. Now, we know from Ball and Yeo (1993) (see theorem A.2 of the appendix) that the second marginal $\left(\sigma_{t}\right)_{t \geq 0}$ of the dynamics is a Markov jump process with rates $c\left(\sigma, \sigma^{\prime}\right)$ if and only if for any $\eta \in\{0,1\}^{E}, \sigma, \sigma^{\prime} \in\{-1,1\}^{V}$, the sum $\sum_{\eta^{\prime} \in\{0,1\}^{E}} q\left((\eta, \sigma),\left(\eta^{\prime}, \sigma^{\prime}\right)\right)$ does not depend on $\eta$. We have then

$$
\forall \eta \in\{0,1\}^{E} \quad \forall \sigma, \sigma^{\prime} \in\{-1,1\}^{V} \quad \sum_{\eta^{\prime} \in\{0,1\}^{E}} q\left((\eta, \sigma),\left(\eta^{\prime}, \sigma^{\prime}\right)\right)=c\left(\sigma, \sigma^{\prime}\right) .
$$


Suppose that this is the case. The second marginal $\left(\sigma_{t}\right)_{t>0}$ will then be the Glauber dynamics with the transition rates (3.4) as soon as the sum in (5.4) is equal, up to some positive multiplicative constant, to the transitions rates given in (3.4). More precisely, we should have, up to some positive multiplicative constant,

$$
c\left(\sigma, \sigma^{\prime}\right)=\left\{\begin{array}{cc}
(1-p)^{\left|\left\{e \in E_{x}, \delta_{\sigma}(e)=1\right\}\right|} & \text { if } \sigma^{\prime}=\sigma^{x} \\
-\sum_{x \in V}(1-p)^{\left|\left\{e \in E_{x}, \delta_{\sigma}(e)=1\right\}\right|} & \text { if } \sigma^{\prime}=\sigma \\
0 & \text { otherwise }
\end{array}\right.
$$

where $E_{x}$ denotes the set of the edges $e$ in $E$ having $x$ as endvertex. Ideally, we would also wish that the first marginal $\left(\eta_{t}\right)_{t \geq 0}$ is a Markov jump process with rates $\tilde{c}\left(\eta, \eta^{\prime}\right)$. This will be the case if and only if, for any $\eta \in\{0,1\}^{E}, \eta^{\prime} \in\{0,1\}^{E}$, $\sigma \in\{-1,1\}^{V}$, the sum $\sum_{\sigma^{\prime} \in\{-1,1\}^{V}} q\left((\eta, \sigma),\left(\eta^{\prime}, \sigma^{\prime}\right)\right)$ does not depend on $\sigma$. We would have then

$$
\forall \eta, \eta^{\prime} \in\{0,1\}^{E} \quad \forall \sigma \in\{-1,1\}^{V} \quad \sum_{\sigma^{\prime} \in\{-1,1\}^{V}} q\left((\eta, \sigma),\left(\eta^{\prime}, \sigma^{\prime}\right)\right)=\tilde{c}\left(\eta, \eta^{\prime}\right) .
$$

So, to sum up, we dream of constructing a Markov process on $\{0,1\}^{E} \times\{-1,1\}^{V}$ with transition rates satisfying (5.1), (5.2), (5.3), (5.4), (5.5) and (5.6), that is a Markov jump process $\left(\eta_{t}, \sigma_{t}\right)_{t \geq 0}$ which is reversible with respect to the $I P$ measure, whose marginal on the spins is a Glauber dynamics, and whose marginal on the edges is Markovian.

\section{One change at a time}

We present here our first try. We build a simple dynamics which updates at most one site or one edge at each time, which is reversible with respect to the coupling measure $I P$ and whose marginal on the spins has the same rates as a Glauber dynamics, although it is not Markovian. Recall that, for $x \in V, E_{x}$ is the set of the edges $e$ in $E$ having $x$ as endvertex, i.e.,

$$
E_{x}=\{e=\langle x, y\rangle \in E: y \in V\} .
$$

For $\sigma \in\{-1,1\}^{V}$ and $x \in V, \sigma^{x}$ is the element of $\{-1,1\}^{V}$ obtained from $\sigma$ by reversing the spin at $x$, i.e.,

$$
\sigma^{x}(x)=-\sigma(x), \quad \forall y \in V \backslash\{x\} \quad \sigma^{x}(y)=\sigma(y) .
$$

For $\eta \in\{0,1\}^{E}$ and $e \in E$, we denote by $\eta^{e}$ the element of $\{0,1\}^{E}$ obtained from $\eta$ by changing the value of $\eta(e)$, i.e.,

$$
\eta^{e}(e)=1-\eta(e), \quad \forall f \in E \backslash\{e\} \quad \eta^{e}(f)=\eta(f) .
$$

Before introducing the transition rates of the one change dynamics, we give a condition that the transition rates have to fulfill in order to have the properties announced at the beginning of the section.

Lemma 6.1. Let $\left(\eta_{t}, \sigma_{t}\right)_{t \geq 0}$ be a Markov jump process, which updates at most one site or one edge at each time and which is reversible with respect to the coupling measure IP. Let us denote by $q\left((\eta, \sigma),\left(\eta^{\prime}, \sigma^{\prime}\right)\right)$ the transition rates of $\left(\eta_{t}, \sigma_{t}\right)_{t \geq 0}$. Let $(\eta, \sigma)$ be a pair of compatible configurations. If there exists $x \in V$ such that $q\left((\eta, \sigma),\left(\eta, \sigma^{x}\right)\right) \neq 0$, then all the edges of $E_{x}$ are closed in $\eta$. 
Proof: The detailed balance equation (5.3) yields that, for any $\eta \in\{0,1\}, \sigma \in$ $\{-1,1\}^{V}$ and any $x \in V$,

$$
\operatorname{IP}(\eta, \sigma) q\left((\eta, \sigma),\left(\eta, \sigma^{x}\right)\right)=I P\left(\eta, \sigma^{x}\right) q\left(\left(\eta, \sigma^{x}\right),(\eta, \sigma)\right),
$$

which gives, thanks to (2.1),

$$
\mathbb{1}_{(\eta, \sigma) \in \mathcal{C}} q\left((\eta, \sigma),\left(\eta, \sigma^{x}\right)\right)=\mathbb{1}_{\left(\eta, \sigma^{x}\right) \in \mathcal{C}} q\left(\left(\eta, \sigma^{x}\right),(\eta, \sigma)\right) .
$$

Since $(\eta, \sigma)$ are supposed to be compatible, the last equality implies that

$$
q\left((\eta, \sigma),\left(\eta, \sigma^{x}\right)\right)=q\left(\left(\eta, \sigma^{x}\right),(\eta, \sigma)\right) \prod_{e \in E_{x}} \mathbb{1}_{\eta(e) \leq 1-\delta_{\sigma}(e)} .
$$

Consequently, if $q\left((\eta, \sigma),\left(\eta, \sigma^{x}\right)\right) \neq 0$ then necessarily $\eta(e) \leq 1-\delta_{\sigma}(e)$ for any $e \in E_{x}$. Since the configurations $\eta$ and $\sigma$ are compatible, necessarily $\eta(e)=0$ for any $e \in E_{x}$.

We define next the transition rates of our dynamics. This dynamics flips a spin at rate one when all the incident edges are closed and it flips an edge with rate $1-p$ if it is open and with rate $p$ if it is closed and it has the same spins at its endvertices.

Definition 6.2. Let $(\eta, \sigma),\left(\eta^{\prime}, \sigma^{\prime}\right)$ be two elements of $\{0,1\}^{E} \times\{-1,1\}^{V}$. We consider several cases:

- If $\eta^{\prime}=\eta$ and there exists $x \in V$ such that $\sigma^{\prime}=\sigma^{x}, \eta(e)=0$ for any $e \in E_{x}$, then we define $c\left((\eta, \sigma),\left(\eta^{\prime}, \sigma^{\prime}\right)\right)=1$.

- If $\sigma^{\prime}=\sigma$ and there exists $e \in E$ such that $\eta^{\prime}=\eta^{e}$ then we set

$$
c\left((\eta, \sigma),\left(\eta^{\prime}, \sigma^{\prime}\right)\right)=p \mathbb{1}_{\eta(e)=0} \delta_{\sigma}(e)+(1-p) \mathbb{1}_{\eta(e)=1} .
$$

- Otherwise, if $(\eta, \sigma) \neq\left(\eta^{\prime}, \sigma^{\prime}\right)$, then we set $c\left((\eta, \sigma),\left(\eta^{\prime}, \sigma^{\prime}\right)\right)=0$.

- Finally, if $(\eta, \sigma)=\left(\eta^{\prime}, \sigma^{\prime}\right)$, then we set

$$
c((\eta, \sigma),(\eta, \sigma))=-\sum_{\left(\eta^{\prime}, \sigma^{\prime}\right),\left(\eta^{\prime}, \sigma^{\prime}\right) \neq(\eta, \sigma)} c\left((\eta, \sigma),\left(\eta^{\prime}, \sigma^{\prime}\right)\right) .
$$

Proposition 6.3. The transition rates introduced in definition 6.2 satisfy the detailed balance equation (5.3).

Proof: We check first that, for any $(\eta, \sigma)$ in $\{0,1\}^{E} \times\{-1,1\}^{V}$ and $e \in E$,

$$
I P(\eta, \sigma) c\left((\eta, \sigma),\left(\eta^{e}, \sigma\right)\right)=I P\left(\eta^{e}, \sigma\right) c\left(\left(\eta^{e}, \sigma\right),(\eta, \sigma)\right) .
$$

Let $(\eta, \sigma)$ in $\{0,1\}^{E} \times\{-1,1\}^{V}$ and $e \in E$ be fixed. We have, since $\eta(e)=1-\eta^{e}(e)$,

$$
\begin{aligned}
& \left(p \mathbb{I}_{\eta(e)=1} \delta_{\sigma}(e)+(1-p) \mathbb{I}_{\eta(e)=0}\right) \times\left(p \mathbb{I}_{\eta(e)=0} \delta_{\sigma}(e)+(1-p) \mathbb{I}_{\eta(e)=1}\right) \\
& =\left(p \mathbb{I}_{\eta^{e}(e)=1} \delta_{\sigma}(e)+(1-p) \mathbb{I}_{\eta^{e}(e)=0}\right) \times\left(p \mathbb{I}_{\eta^{e}(e)=0} \delta_{\sigma}(e)+(1-p) \mathbb{I}_{\eta^{e}(e)=1}\right) .
\end{aligned}
$$

We also have, since $\eta^{e}=\eta$ on $E \backslash\{e\}$,

$$
\begin{aligned}
\prod_{f \in E \backslash\{e\}}\left(p \mathbb{I}_{\eta(f)=1} \delta_{\sigma}(f)+(1-p) \mathbb{I}_{\eta(f)=0}\right) & \\
& =\prod_{f \in E \backslash\{e\}}\left(p \mathbb{I}_{\eta^{e}(f)=1} \delta_{\sigma}(f)+(1-p) \mathbb{I}_{\eta^{e}(f)=0}\right) .
\end{aligned}
$$


Noting that

$$
\begin{aligned}
& I P(\eta, \sigma)= \\
& \quad \frac{1}{Z}\left(p \mathbb{1}_{\eta(e)=1} \delta_{\sigma}(e)+(1-p) \mathbb{1}_{\eta(e)=0}\right) \prod_{f \in E \backslash\{e\}}\left(p \mathbb{I}_{\eta(f)=1} \delta_{\sigma}(f)+(1-p) \mathbb{1}_{\eta(f)=0}\right),
\end{aligned}
$$

we get (6.1) by multiplying each side of (6.3) by (6.2) and by dividing by $Z$. We now prove that, for any $(\eta, \sigma)$ in $\{0,1\}^{E} \times\{-1,1\}^{V}$ and any $x \in V$,

$$
I P(\eta, \sigma) c\left((\eta, \sigma),\left(\eta, \sigma^{x}\right)\right)=I P\left(\eta, \sigma^{x}\right) c\left(\left(\eta, \sigma^{x}\right),(\eta, \sigma)\right)
$$

Noting that

$$
c\left((\eta, \sigma),\left(\eta, \sigma^{x}\right)\right)=\prod_{e \in E_{x}} \mathbb{1}_{\eta(e)=0}
$$

and that $\delta_{\sigma}(e)=\delta_{\sigma^{x}}(e)$ for any $e \in E \backslash E_{x}$, we deduce that

$$
\begin{aligned}
& c\left((\eta, \sigma),\left(\eta, \sigma^{x}\right)\right) I P(\eta, \sigma) \\
& =\frac{1}{Z}\left(\prod_{e \in E_{x}} \mathbb{1}_{\eta(e)=0}\right)\left(\prod_{e \in E}\left(p \mathbb{I}_{\eta(e)=1} \delta_{\sigma}(e)+(1-p) \mathbb{I}_{\eta(e)=0}\right)\right) \\
& =\frac{1}{Z}\left(\prod_{e \in E_{x}}\left((1-p) \mathbb{1}_{\eta(e)=0}\right)\right)\left(\prod_{e \in E \backslash E_{x}}\left(p \mathbb{I}_{\eta(e)=1} \delta_{\sigma}(e)+(1-p) \mathbb{I}_{\eta(e)=0}\right)\right) \\
& =\frac{1}{Z}\left(\prod_{e \in E_{x}}\left((1-p) \mathbb{1}_{\eta(e)=0}\right)\right)\left(\prod_{e \in E \backslash E_{x}}\left(p \mathbb{I}_{\eta(e)=1} \delta_{\sigma^{x}}(e)+(1-p) \mathbb{I}_{\eta(e)=0}\right)\right) \\
& =\frac{1}{Z}\left(\prod_{e \in E_{x}} \mathbb{I}_{\eta(e)=0}\right)\left(\prod_{e \in E}\left(p \mathbb{I}_{\eta(e)=1} \delta_{\sigma^{x}}(e)+(1-p) \mathbb{I}_{\eta(e)=0}\right)\right) \\
& =c\left(\left(\eta, \sigma^{x}\right),(\eta, \sigma)\right) I P\left(\eta, \sigma^{x}\right) .
\end{aligned}
$$

This finishes the proof of condition (6.4). Since the transition rates $c\left((\eta, \sigma),\left(\eta^{\prime}, \sigma^{\prime}\right)\right)$ given in definition 6.2 allow to perform at most one change at a time, the reversibility condition reduces to the two conditions (6.1) and (6.4), so the proof of Proposition 6.3 is complete.

The following proposition shows that, if the initial condition $\left(\eta_{0}, \sigma_{0}\right)$ is distributed as $I P$, then the infinitesimal behavior of the marginal on the spins of the process $\left(\eta_{t}, \sigma_{t}\right)_{t \geq 0}$, whose rates are introduced in definition 6.2 , is the same as the transition rates given in (5.5).

Proposition 6.4. Let $\left(\eta_{t}, \sigma_{t}\right)_{t \geq 0}$ be a Markov jump process with the transition rates introduced in definition 6.2. Suppose that the pair $\left(\eta_{0}, \sigma_{0}\right)$ is distributed according to $I P$. Then, for any $x \in V$, for any $\sigma \in\{-1,1\}^{V}$ and any $s \geq 0$,

$$
\lim _{t \rightarrow 0} \frac{1}{t} \mathbb{P}\left(\sigma_{t+s}=\sigma^{x} \mid \sigma_{s}=\sigma\right)=(1-p)^{\left|\left\{e \in E_{x}, \delta_{\sigma}(e)=1\right\}\right|} .
$$


Proof: We have, for any $\sigma \in\{-1,1\}^{V}, x \in V$ and $s \geq 0$,

$$
\begin{aligned}
& \mathbb{P}\left(\sigma_{t+s}=\sigma^{x} \mid \sigma_{s}=\sigma\right) \\
& =\sum_{\eta \in\{0,1\}^{E}} \sum_{\eta^{\prime} \in\{0,1\}^{E}} \mathbb{P}\left(\sigma_{t+s}=\sigma^{x}, \eta_{t+s}=\eta^{\prime}, \eta_{s}=\eta \mid \sigma_{s}=\sigma\right) \\
& =\sum_{\eta \in\{0,1\}^{E}} \sum_{\eta^{\prime} \in\{0,1\}^{E}} \mathbb{P}\left(\sigma_{t+s}=\sigma^{x}, \eta_{t+s}=\eta^{\prime} \mid \sigma_{s}=\sigma, \eta_{s}=\eta\right) \mathbb{P}\left(\eta_{s}=\eta \mid \sigma_{s}=\sigma\right) .
\end{aligned}
$$

Since $\left(\eta_{t}, \sigma_{t}\right)_{t \geq 0}$ is a Markov jump process with the transition rates $c\left((\eta, \sigma),\left(\eta^{\prime}, \sigma^{\prime}\right)\right)$ introduced in definition 6.2 , then

$$
\begin{aligned}
\lim _{t \rightarrow 0} \frac{1}{t} \mathbb{P}\left(\sigma_{t+s}=\sigma^{x}, \eta_{t+s}=\eta^{\prime} \mid \sigma_{s}\right. & \left.=\sigma, \eta_{s}=\eta\right) \\
& =c\left((\eta, \sigma),\left(\eta^{\prime}, \sigma^{x}\right)\right)=\mathbb{1}_{\eta^{\prime}=\eta} \prod_{e \in E_{x}} \mathbb{1}_{\eta(e)=0 .} .
\end{aligned}
$$

Hence we get, combining (6.6) and (6.7), for any $s \geq 0$,

$$
\lim _{t \rightarrow 0} \frac{1}{t} \mathbb{P}\left(\sigma_{t+s}=\sigma^{x} \mid \sigma_{s}=\sigma\right)=\sum_{\eta \in\{0,1\}^{E}}\left(\prod_{e \in E_{x}} \mathbb{1}_{\eta(e)=0}\right) \mathbb{P}\left(\eta_{s}=\eta \mid \sigma_{s}=\sigma\right) .
$$

The measure $I P$ is, by proposition 6.3 , a reversible and a stationary measure for the process $\left(\eta_{t}, \sigma_{t}\right)_{t \geq 0}$. Hence, if $\left(\eta_{0}, \sigma_{0}\right)$ is distributed as $I P$, then, for any $s \geq 0$, $\left(\eta_{s}, \sigma_{s}\right)$ is also distributed as $I P$. Consequently, under the hypothesis of proposition 6.4 , we have

$$
\mathbb{P}\left(\eta_{s}=\eta \mid \sigma_{s}=\sigma\right)=\frac{\mathbb{P}\left(\eta_{s}=\eta, \sigma_{s}=\sigma\right)}{\mathbb{P}\left(\sigma_{s}=\sigma\right)}=\frac{I P(\eta, \sigma)}{\sum_{\eta^{\prime} \in\{0,1\}^{E}} I P\left(\eta^{\prime}, \sigma\right)} .
$$

We conclude that

$$
\begin{aligned}
& \lim _{t \rightarrow 0} \frac{1}{t} \mathbb{P}\left(\sigma_{t+s}=\sigma^{x} \mid \sigma_{s}=\sigma\right) \\
& =\frac{\sum_{\eta \in\{0,1\}^{E}, \eta \equiv 0 \text { on } E_{x}} I P(\eta, \sigma)}{\sum_{\eta^{\prime} \in\{0,1\}^{E}} I P\left(\eta^{\prime}, \sigma\right)}
\end{aligned}
$$

We compute these sums, starting with the very definition of the coupling measure $I P$. We have

$$
\sum_{\eta \in\{0,1\}^{E}, \eta \equiv 0 \text { on } E_{x}} I P(\eta, \sigma)=\frac{1}{Z}(1-p)^{\left|\left\{e \in E: \delta_{\sigma}(e)=0\right\}\right|}(1-p)^{\left|\left\{e \in E_{x}: \delta_{\sigma}(e)=1\right\}\right|} .
$$

The sum in the denominator has already been computed in equation (2.3). Equations (2.3), (6.8) and (6.9) together yield the desired result (6.5).

We consider next the marginal on the edges and we obtain a similar result. Recall that the quantity $\gamma_{\eta}(e)$ has been defined in section 4.

Proposition 6.5. Let $\left(\eta_{t}, \sigma_{t}\right)_{t \geq 0}$ be a Markov jump process with the transition rates introduced in definition 6.2. Suppose that the pair $\left(\eta_{0}, \sigma_{0}\right)$ is distributed according to IP. Then, for any $e \in E$, for any $\eta \in\{0,1\}^{E}$ and any $s \geq 0$,

$$
\lim _{t \rightarrow 0} \frac{1}{t} \mathbb{P}\left(\eta_{t+s}=\eta^{e} \mid \eta_{s}=\eta\right)=(1-p) \mathbb{I}_{\eta(e)=1}+p \mathbb{I}_{\eta(e)=0, \gamma_{\eta}(e)=1}+\frac{p}{2} \mathbb{I}_{\eta(e)=0, \gamma_{\eta}(e)=0} .
$$


Proof: We have, for any $\eta \in\{0,1\}^{E}, e \in E$ and $s \geq 0$,

$$
\begin{aligned}
& \mathbb{P}\left(\eta_{t+s}=\eta^{e} \mid \eta_{s}=\eta\right)= \\
& \sum_{\sigma \in\{-1,1\}^{V}} \sum_{\sigma^{\prime} \in\{-1,1\}^{V}} \mathbb{P}\left(\sigma_{t+s}=\sigma^{\prime}, \eta_{t+s}=\eta^{e}, \sigma_{s}=\sigma \mid \eta_{s}=\eta\right) \\
& =\sum_{\sigma \in\{-1,1\}^{V}} \sum_{\sigma^{\prime} \in\{-1,1\}^{V}} \mathbb{P}\left(\sigma_{t+s}=\sigma^{\prime}, \eta_{t+s}=\eta^{e} \mid \sigma_{s}=\sigma, \eta_{s}=\eta\right) \mathbb{P}\left(\sigma_{s}=\sigma \mid \eta_{s}=\eta\right) .
\end{aligned}
$$

Since $\left(\eta_{t}, \sigma_{t}\right)_{t \geq 0}$ is a Markov jump process with the transition rates $c\left((\eta, \sigma),\left(\eta^{\prime}, \sigma^{\prime}\right)\right)$ introduced in definition 6.2, then

$$
\begin{aligned}
\lim _{t \rightarrow 0} \frac{1}{t} \mathbb{P}\left(\sigma_{t+s}=\sigma^{\prime}, \eta_{t+s}=\eta^{e} \mid \sigma_{s}=\sigma, \eta_{s}=\eta\right)=c\left((\eta, \sigma),\left(\eta^{e}, \sigma^{\prime}\right)\right) \\
=\mathbb{I}_{\sigma^{\prime}=\sigma}\left(p \mathbb{1}_{\eta(e)=0} \delta_{\sigma}(e)+(1-p) \mathbb{I}_{\eta(e)=1}\right) .
\end{aligned}
$$

Combining (6.11) and (6.12), we get, for any $s \geq 0$,

$$
\begin{aligned}
\lim _{t \rightarrow 0} \frac{1}{t} \mathbb{P}\left(\eta_{t+s}=\eta^{e} \mid \eta_{s}=\eta\right)= & \\
& \sum_{\sigma \in\{-1,1\}^{V}}\left(p \mathbb{I}_{\eta(e)=0} \delta_{\sigma}(e)+(1-p) \mathbb{I}_{\eta(e)=1}\right) \mathbb{P}\left(\sigma_{s}=\sigma \mid \eta_{s}=\eta\right) .
\end{aligned}
$$

The measure $I P$ is, by proposition 6.3 , a reversible and a stationary measure for the process $\left(\eta_{t}, \sigma_{t}\right)_{t \geq 0}$. Hence, if $\left(\eta_{0}, \sigma_{0}\right)$ is distributed as $I P$, then, for any $s \geq 0$, $\left(\eta_{s}, \sigma_{s}\right)$ is also distributed as $I P$. Consequently, under the hypothesis of proposition 6.5 , we have

$$
\mathbb{P}\left(\sigma_{s}=\sigma \mid \eta_{s}=\eta\right)=\frac{\mathbb{P}\left(\eta_{s}=\eta, \sigma_{s}=\sigma\right)}{\mathbb{P}\left(\eta_{s}=\eta\right)}=\frac{I P(\eta, \sigma)}{\sum_{\sigma^{\prime} \in\{-1,1\}^{V}} I P\left(\eta, \sigma^{\prime}\right)} .
$$

We conclude that

$$
\begin{aligned}
\lim _{t \rightarrow 0} \frac{1}{t} \mathbb{P}\left(\eta_{t+s}=\eta^{e} \mid \eta_{s}=\eta\right)= & \\
& \frac{\sum_{\sigma \in\{-1,1\}^{V}}\left(p \mathbb{1}_{\eta(e)=0} \delta_{\sigma}(e)+(1-p) \mathbb{I}_{\eta(e)=1}\right) I P(\eta, \sigma)}{\sum_{\sigma^{\prime} \in\{-1,1\}^{V}} I P\left(\eta, \sigma^{\prime}\right)} .
\end{aligned}
$$

We compute these sums, starting with the very definition of the coupling measure $I P$. For a configuration $\eta$ such that $\gamma_{\eta}(e)=1$, we have

$$
\sum_{\sigma \in\{-1,1\}^{V}} \delta_{\sigma}(e) I P(\eta, \sigma)=\sum_{\sigma \in\{-1,1\}^{V}} I P(\eta, \sigma)=\Phi_{p, 2}(\eta) .
$$

For a configuration $\eta$ such that $\gamma_{\eta}(e)=0$, we have

$$
\sum_{\sigma \in\{-1,1\}^{V}} \delta_{\sigma}(e) I P(\eta, \sigma)=\sum_{\sigma \in\{-1,1\}^{V}} \frac{1}{2}(1+\sigma(x) \sigma(y)) I P(\eta, \sigma)=\frac{1}{2} \Phi_{p, 2}(\eta) .
$$

From the previous identities, we obtain that

$$
\begin{aligned}
\sum_{\sigma \in\{-1,1\}^{V}} & \left(p \mathbb{I}_{\eta(e)=0} \delta_{\sigma}(e)+(1-p) \mathbb{I}_{\eta(e)=1}\right) I P(\eta, \sigma)= \\
& \left(p \mathbb{I}_{\eta(e)=0, \gamma_{\eta}(e)=1}+\frac{p}{2} \mathbb{I}_{\eta(e)=0, \gamma_{\eta}(e)=0}+(1-p) \mathbb{I}_{\eta(e)=1}\right) \Phi_{p, 2}(\eta) .
\end{aligned}
$$


The sum in the denominator has already been computed in equation (2.2), it is equal to $\Phi_{p, 2}(\eta)$. Equations (2.2), (6.13) and (6.14) together yield the desired result (6.10).

Although the infinitesimal behavior of the marginal on the spins of $\left(\eta_{t}, \sigma_{t}\right)_{t \geq 0}$ is the same as for the Glauber dynamics with the transition rates given in (5.5), the process $\left(\sigma_{t}\right)_{t \geq 0}$ does not evolve according to a Glauber dynamics, because it is not a Markovian process. Similarly, the marginal on the edges is not a Markovian process. We summarize these results in the following theorem.

Theorem 6.6. We suppose that the set of edges $E$ is not empty. Let $\left(\eta_{t}, \sigma_{t}\right)_{t \geq 0}$ be a Markov jump process with the transition rates introduced in definition 6.2. Then $\left(\eta_{t}, \sigma_{t}\right)_{t \geq 0}$ is reversible with respect to the coupling measure IP. However its two marginal processes $\left(\eta_{t}\right)_{t \geq 0}$ and $\left(\sigma_{t}\right)_{t \geq 0}$ are not Markovian jump processes.

Proof: The reversibility property has been proved in proposition 6.3. Yet the sum

$$
\sum_{\eta^{\prime} \in\{0,1\}^{E}} c\left((\eta, \sigma),\left(\eta^{\prime}, \sigma^{x}\right)\right)
$$

depends on $\eta$ for some $\sigma \in\{-1,1\}^{V}$ and $x \in V$. In fact, we have, for any $\sigma \in$ $\{-1,1\}^{V}$ and $x \in V$,

$$
\sum_{\eta^{\prime} \in\{0,1\}^{E}} c\left((\eta, \sigma),\left(\eta^{\prime}, \sigma^{x}\right)\right)=c\left((\eta, \sigma),\left(\eta, \sigma^{x}\right)\right)=\prod_{e \in E_{x}} \mathbb{1}_{\eta(e)=0} .
$$

Similarly, the sum

$$
\sum_{\sigma^{\prime} \in\{-1,1\}^{V}} c\left((\eta, \sigma),\left(\eta^{e}, \sigma^{\prime}\right)\right)
$$

depends on $\sigma$ for some $\eta \in\{0,1\}^{E}$ and $e \in E$. In fact, we have, for any $\eta \in\{0,1\}^{E}$ and $e \in E$,

$$
\sum_{\sigma^{\prime} \in\{-1,1\}^{V}} c\left((\eta, \sigma),\left(\eta^{e}, \sigma^{\prime}\right)\right)=c\left((\eta, \sigma),\left(\eta^{e}, \sigma\right)\right)=p \mathbb{I}_{\eta(e)=0} \delta_{\sigma}(e)+(1-p) \mathbb{I}_{\eta(e)=1} .
$$

The proof of Theorem 6.6 is complete thanks to Theorem 3.1 in Ball and Yeo (1993) (see theorem A.2 in the appendix).

Theorem 6.6 shows that the Markov jump process with the transition rates given in definition 6.2 does not fulfill our dream, which was to build a Markov jump process on $\{0,1\}^{E} \times\{-1,1\}^{V}$, reversible with respect to the IP measure, with a Glauber dynamics as the marginal on the spins and such that the marginal on the edges is Markovian. The following theorem shows that there is no hope to realize this dream with a Markov jump process $\left(\eta_{t}, \sigma_{t}\right)_{t \geq 0}$ which updates at most one site or one edge at each time.

Theorem 6.7. Let $\left(\eta_{t}, \sigma_{t}\right)_{t \geq 0}$ be a Markov jump process which updates at most one site or one edge at each time and whose marginal on the spins is a Markov jump process with transition rates satisfying (3.1). Then $\left(\eta_{t}, \sigma_{t}\right)_{t \geq 0}$ cannot be reversible with respect to the coupling measure IP. 
Proof: Let $\left(\eta_{t}, \sigma_{t}\right)_{t>0}$ be a Markov jump process satisfying the assumptions of theorem 6.7 and let $q\left((\eta, \sigma),\left(\eta^{\prime}, \sigma^{\prime}\right)\right)$ be its transition rates. Since this Markov process updates at most one site or one edge at each time, we have

$$
\sum_{\eta^{\prime} \in\{0,1\}^{E}} q\left((\eta, \sigma),\left(\eta^{\prime}, \sigma^{x}\right)\right)=q\left((\eta, \sigma),\left(\eta, \sigma^{x}\right)\right) .
$$

Since the marginal on the spins of $\left(\eta_{t}, \sigma_{t}\right)_{t \geq 0}$ is a Markov process with transition rates $c\left(\sigma, \sigma^{\prime}\right)$ satisfying (3.1), Theorem 3.1 in Ball and Yeo (1993) (see theorem A.2 in the appendix) gives that

$$
\left.\forall \eta \in\{0,1\}^{E} \quad \sum_{\eta^{\prime} \in\{0,1\}^{E}} q\left((\eta, \sigma),\left(\eta^{\prime}, \sigma^{x}\right)\right)\right)=c\left(\sigma, \sigma^{x}\right) .
$$

Hence, for any $\eta \in\{0,1\}^{E}$, any $\sigma \in\{-1,1\}^{V}$ and any $x \in V$

$$
q\left((\eta, \sigma),\left(\eta, \sigma^{x}\right)\right)=c\left(\sigma, \sigma^{x}\right) .
$$

Suppose now that the detailed balance equation (5.3) is satisfied. We deduce then from (3.1) and lemma 6.1 that necessarily $\eta(e)=0$ for $e \in E_{x}$. We conclude that the detailed balance equation (5.3) is not satisfied for every $\eta \in\{0,1\}^{E}$ and every $\sigma \in\{-1,1\}^{V}$. This completes the proof.

Theorem 3.2 in Ball and Yeo (1993) (see theorem A.3 in the appendix) implies that, for a process like the above one, in which at most one marginal process moves at each time step, the marginal processes are Markovian if and only if they are independent. If that were the case, then the equilibrium measure would be a product measure. Therefore it is impossible to realize our dream with a process which changes only one edge or one spin at a time. In the next section, we construct a process which can change simultaneously one spin and its incident edges.

\section{One site and the incident edges}

We build here a dynamics which updates at most one site and its incident edges at each time, which is reversible with respect to the coupling measure $I P$ and whose marginal on the spins is a Glauber dynamics. For $x \in V$, recall that $E_{x}$ is the set of the edges $e$ in $E$ having $x$ as endvertex, i.e.,

$$
E_{x}=\{e \in E, e=\langle x, y\rangle, y \in V\}
$$

and define

$$
\mathcal{C}_{x}=\left\{(\eta, \sigma) \in\{0,1\}^{E} \times\{-1,1\}^{V}: \eta(e) \leq \delta_{\sigma}(e) \text { for any } e \in E_{x}\right\} .
$$

The following definition gives the transition rates of the dynamics. This dynamics flips spins whose open incident edges have the same spins at their endvertices.

Definition 7.1. Let $(\eta, \sigma),\left(\eta^{\prime}, \sigma^{\prime}\right)$ be two elements of $\{0,1\}^{E} \times\{-1,1\}^{V}$. We consider several cases:

- If there exists $x \in V$ such that $\sigma^{\prime}=\sigma^{x}$ and $\eta^{\prime}(e)=\eta(e)$ for any $e \in E \backslash E_{x}$, then we define

$$
c\left((\eta, \sigma),\left(\eta^{\prime}, \sigma^{\prime}\right)\right)=(1-p)^{\left|\left\{e \in E_{x}, \eta^{\prime}(e)=0\right\}\right|} p^{\left|\left\{e \in E_{x}, \eta^{\prime}(e)=1\right\}\right|} \mathbb{I}_{\left(\eta^{\prime}, \sigma^{\prime}\right) \in \mathcal{C}_{x}} .
$$

- Otherwise, if $(\eta, \sigma) \neq\left(\eta^{\prime}, \sigma^{\prime}\right)$, then we set $c\left((\eta, \sigma),\left(\eta^{\prime}, \sigma^{\prime}\right)\right)=0$. 
- Finally, if $(\eta, \sigma)=\left(\eta^{\prime}, \sigma^{\prime}\right)$, then we set

$$
c((\eta, \sigma),(\eta, \sigma))=-\sum_{\left(\eta^{\prime}, \sigma^{\prime}\right),\left(\eta^{\prime}, \sigma^{\prime}\right) \neq(\eta, \sigma)} c\left((\eta, \sigma),\left(\eta^{\prime}, \sigma^{\prime}\right)\right) .
$$

Proposition 7.2. The transition rates introduced in definition 7.1 satisfy the detailed balance equation (5.3).

Proof: Let $(\eta, \sigma)$ and $\left(\eta^{\prime}, \sigma^{\prime}\right)$ be fixed in $\{0,1\}^{E} \times\{-1,1\}^{V}$. We have to prove (5.3) only in the case where there exists $x \in V$ for which

$$
\sigma^{\prime}=\sigma^{x}, \quad \forall e \in E \backslash E_{x} \quad \eta^{\prime}(e)=\eta(e),
$$

because in all the other cases, the detailed balance equation (5.3) is trivially satisfied. Let us fix $x \in V$. Suppose first that $(\eta, \sigma)$ and $\left(\eta^{\prime}, \sigma^{\prime}\right)$ are two elements of $\mathcal{C}_{x}$ satisfying (7.1). We get then, for $e \in E_{x}$,

$$
\mathbb{1}_{\eta(e)=1} \delta_{\sigma}(e)=\mathbb{1}_{\eta(e)=1}, \quad \mathbb{1}_{\eta^{\prime}(e)=1} \delta_{\sigma^{x}}(e)=\mathbb{1}_{\eta^{\prime}(e)=1},
$$

therefore

$$
\begin{aligned}
\left(\prod_{e \in E_{x}}\right. & \left.\left(p \mathbb{I}_{\eta(e)=1} \delta_{\sigma}(e)+(1-p) \mathbb{I}_{\eta(e)=0}\right)\right) c\left((\eta, \sigma),\left(\eta^{\prime}, \sigma^{x}\right)\right) \\
= & p^{\left|\left\{e \in E_{x}, \eta(e)=1\right\}\right|}(1-p)^{\left|\left\{e \in E_{x}, \eta(e)=0\right\}\right|}(1-p)^{\left|\left\{e \in E_{x}, \eta^{\prime}(e)=0\right\}\right|} p^{\left|\left\{e \in E_{x}, \eta^{\prime}(e)=1\right\}\right|} \\
\quad & \left(\prod_{e \in E_{x}}\left(p \mathbb{I}_{\eta^{\prime}(e)=1} \delta_{\sigma^{x}}(e)+(1-p) \mathbb{I}_{\eta^{\prime}(e)=0}\right)\right) c\left(\left(\eta^{\prime}, \sigma^{x}\right),(\eta, \sigma)\right) .
\end{aligned}
$$

Now we multiply each member of the last equality by

$$
\begin{aligned}
& \prod_{e \in E \backslash E_{x}}\left(p \mathbb{I}_{\eta(e)=1} \delta_{\sigma}(e)+(1-p) \mathbb{1}_{\eta(e)=0}\right) \\
= & \prod_{e \in E \backslash E_{x}}\left(p \mathbb{1}_{\eta^{\prime}(e)=1} \delta_{\sigma^{x}}(e)+(1-p) \mathbb{I}_{\eta^{\prime}(e)=0}\right)
\end{aligned}
$$

and we obtain the detailed balance equation (5.3). Suppose now that $(\eta, \sigma) \in \mathcal{C}_{x}$ and $\left(\eta^{\prime}, \sigma^{x}\right) \notin \mathcal{C}_{x}$. We have then, by the definition of the transition rates,

$$
I P(\eta, \sigma) c\left((\eta, \sigma),\left(\eta^{\prime}, \sigma^{x}\right)\right)=0 .
$$

Moreover $I P\left(\eta^{\prime}, \sigma^{x}\right)=0$ because $\left(\eta^{\prime}, \sigma^{x}\right) \notin \mathcal{C}$, therefore

$$
I P\left(\eta^{\prime}, \sigma^{x}\right) c\left(\left(\eta^{\prime}, \sigma^{x}\right),(\eta, \sigma)\right)=0 .
$$

So here again equation (5.3) is satisfied. Finally suppose that $(\eta, \sigma) \notin \mathcal{C}_{x}$. Then $I P(\eta, \sigma)=0$ and so $I P(\eta, \sigma) c\left((\eta, \sigma),\left(\eta^{\prime}, \sigma^{x}\right)\right)=0$ which is equal to the quantity IP $\left(\eta^{\prime}, \sigma^{x}\right) c\left(\left(\eta^{\prime}, \sigma^{x}\right),(\eta, \sigma)\right)$, since the transition rates $c\left(\left(\eta^{\prime}, \sigma^{x}\right),(\eta, \sigma)\right)$ vanish as soon as $(\eta, \sigma) \notin \mathcal{C}_{x}$. We conclude that the detailed balance equation (5.3) is always satisfied.

We compute next the marginal dynamics on the spins.

Proposition 7.3. For any $\eta$ in $\{0,1\}^{E}$ and any $\sigma, \sigma^{\prime}$ in $\{-1,1\}^{V}$, the sum

$$
\sum_{\eta^{\prime} \in\{0,1\}^{E}} c\left((\eta, \sigma),\left(\eta^{\prime}, \sigma^{\prime}\right)\right)
$$


does not depend on $\eta$ and it is equal to

$$
c\left(\sigma, \sigma^{\prime}\right)=\left\{\begin{array}{cc}
(1-p)^{\left|\left\{e \in E_{x}, \delta_{\sigma}(e)=1\right\}\right|} & \text { if } \sigma^{\prime}=\sigma^{x}, \\
-\sum_{x \in V}(1-p)^{\left|\left\{e \in E_{x}, \delta_{\sigma}(e)=1\right\}\right|} & \text { if } \sigma^{\prime}=\sigma, \\
0 & \text { otherwise. }
\end{array}\right.
$$

Proof: Let $\eta \in\{0,1\}^{E}$ be fixed. If $\left|\left\{y \in V, \sigma(y) \neq \sigma^{\prime}(y)\right\}\right| \geq 2$ then, by definition 7.1,

$$
\sum_{\eta^{\prime} \in\{0,1\}^{E}} c\left((\eta, \sigma),\left(\eta^{\prime}, \sigma^{\prime}\right)\right)=0
$$

Suppose now that there exists $x \in V$ such that $\sigma^{\prime}=\sigma^{x}$. Then, by definition 7.1,

$$
\begin{aligned}
& \sum_{\eta^{\prime} \in\{0,1\}^{E}} c\left((\eta, \sigma),\left(\eta^{\prime}, \sigma^{\prime}\right)\right)= \\
& \sum_{\eta^{\prime} \in\{0,1\}^{E}, \eta^{\prime} \equiv \eta \text { on } E \backslash E_{x}}(1-p)^{\left|\left\{e \in E_{x}, \eta^{\prime}(e)=0\right\}\right|} p^{\left|\left\{e \in E_{x}, \eta^{\prime}(e)=1\right\}\right|} \mathbb{I}_{\left(\eta^{\prime}, \sigma^{x}\right) \in \mathcal{C}_{x}} .
\end{aligned}
$$

Our next task is to calculate the last sum. To this end, we use the same proof as for $(2.3)$ (recall that $I P(\eta, \sigma)$ is also given by $(2.1)$ ). We get

$$
\sum_{\eta^{\prime} \in\{0,1\}^{E}} c\left((\eta, \sigma),\left(\eta^{\prime}, \sigma^{x}\right)\right)=(1-p)^{\left|\left\{e \in E_{x}, \delta_{\sigma}(e)=1\right\}\right|} .
$$

Finally, we have to calculate $\sum_{\eta^{\prime} \in\{0,1\}^{E}} c\left((\eta, \sigma),\left(\eta^{\prime}, \sigma\right)\right)$. We have, from definition 7.1,

$$
\begin{aligned}
\sum_{\eta^{\prime} \in\{0,1\}^{E}} c((\eta, \sigma), & \left.\left(\eta^{\prime}, \sigma\right)\right)=c((\eta, \sigma),(\eta, \sigma)) \\
& =-\sum_{\left(\eta^{\prime}, \sigma^{\prime}\right),\left(\eta^{\prime}, \sigma^{\prime}\right) \neq(\eta, \sigma)} c\left((\eta, \sigma),\left(\eta^{\prime}, \sigma^{\prime}\right)\right) \\
& =-\sum_{\eta^{\prime} \in\{0,1\}^{E}} \sum_{x \in V} c\left((\eta, \sigma),\left(\eta^{\prime}, \sigma^{x}\right)\right) \\
& =-\sum_{x \in V} \sum_{\eta^{\prime} \in\{0,1\}^{E}} c\left((\eta, \sigma),\left(\eta^{\prime}, \sigma^{x}\right)\right) \\
& =-\sum_{x \in V}(1-p)^{\left|\left\{e \in E_{x}, \delta_{\sigma}(e)=1\right\}\right|}
\end{aligned}
$$

Equalities (7.2), (7.3) and (7.4) yield the statement of proposition 7.3.

We compute next the marginal dynamics on the edges.

Proposition 7.4. Let $\eta$ and $\eta^{\prime}$ be two different elements of $\{0,1\}^{E}$.

- If there exists $e=\langle x, y\rangle \in E$ such that $\eta^{\prime}(e) \neq \eta(e)$ and $\eta^{\prime} \equiv \eta$ on $E \backslash\{e\}$, then $\sum_{\sigma^{\prime} \in\{-1,1\}^{V}} c\left((\eta, \sigma),\left(\eta^{\prime}, \sigma^{\prime}\right)\right)=\sum_{z \in\{x, y\}}(1-p)^{\left|\left\{e \in E_{z}, \eta^{\prime}(e)=0\right\}\right|} p^{\left|\left\{e \in E_{z}, \eta^{\prime}(e)=1\right\}\right|} \mathbb{I}_{\left(\eta^{\prime}, \sigma^{z}\right) \in \mathcal{C}_{z}}$.

- If there exists $x \in V$ such that $\eta^{\prime} \equiv \eta$ on $E \backslash E_{x}$ and the configurations $\eta, \eta^{\prime}$ differ in more than one edge, then

$$
\sum_{\sigma^{\prime} \in\{-1,1\}^{V}} c\left((\eta, \sigma),\left(\eta^{\prime}, \sigma^{\prime}\right)\right)=(1-p)^{\left|\left\{e \in E_{x}, \eta^{\prime}(e)=0\right\}\right|} p^{\left|\left\{e \in E_{x}, \eta^{\prime}(e)=1\right\}\right|} \mathbb{I}_{\left(\eta^{\prime}, \sigma^{x}\right) \in \mathcal{C}_{x}}
$$


- If for all $x \in V, \eta^{\prime} \neq \eta$ on $E \backslash E_{x}$, then

$$
\sum_{\sigma^{\prime} \in\{-1,1\}^{V}} c\left((\eta, \sigma),\left(\eta^{\prime}, \sigma^{\prime}\right)\right)=0 .
$$

Proof: Let $\eta$ and $\eta^{\prime}$ be two fixed different elements of $\{0,1\}^{E}$. If for all $x \in V, \eta$ and $\eta^{\prime}$ are different on $E \backslash E_{x}$ then we get, by definition 7.1,

$$
\forall \sigma^{\prime} \in\{-1,1\}^{V} \quad c\left((\eta, \sigma),\left(\eta^{\prime}, \sigma^{\prime}\right)\right)=0 .
$$

Consequently,

$$
\sum_{\sigma^{\prime} \in\{-1,1\}^{V}} c\left((\eta, \sigma),\left(\eta^{\prime}, \sigma^{\prime}\right)\right)=0
$$

Suppose now that there exists $x \in V$ such that $\eta \equiv \eta^{\prime}$ on $E \backslash E_{x}$. Let us define the set

We have

$$
\mathcal{V}=\left\{y \in V, \eta \equiv \eta^{\prime} \text { on } E \backslash E_{y}\right\} .
$$

$$
\sum_{\sigma^{\prime} \in\{-1,1\}^{V}} c\left((\eta, \sigma),\left(\eta^{\prime}, \sigma^{\prime}\right)\right)=\sum_{y \in \mathcal{V}} c\left((\eta, \sigma),\left(\eta^{\prime}, \sigma^{y}\right)\right)
$$

Let us set

$$
\mathcal{E}=\left\{f \in E, \eta(f) \neq \eta^{\prime}(f)\right\} .
$$

By hypothesis, this set is not empty and it is a subset of $E_{x}$ because $\eta \neq \eta^{\prime}$ while $\eta \equiv \eta^{\prime}$ on $E \backslash E_{x}$. Suppose first that $\mathcal{E}$ contains only one element, say $\mathcal{E}=\{e\}$. Necessarily one endvertex of $e$ is $x$. Let $y$ be the other endvertex of $e$, so that $e=\langle x, y\rangle$. We have then $\mathcal{V}=\{x, y\}$. Indeed, if there exists a vertex $z$ in $V$ such that

$$
z \neq x \text { and } \eta \equiv \eta^{\prime} \text { on } E \backslash E_{z},
$$

then, since $e \in E_{x}$ and $\eta(e) \neq \eta^{\prime}(e)$, we have also $e \in E_{z}$, whence $e=\langle x, z\rangle$ and $z=y$. Equation (7.6) can now be rewritten as

$$
\sum_{\sigma^{\prime} \in\{-1,1\}^{V}} c\left((\eta, \sigma),\left(\eta^{\prime}, \sigma^{\prime}\right)\right)=c\left((\eta, \sigma),\left(\eta^{\prime}, \sigma^{x}\right)\right)+c\left((\eta, \sigma),\left(\eta^{\prime}, \sigma^{y}\right)\right) .
$$

Suppose now that $\mathcal{E}$ contains more than one element. We claim that, in this case, the set $\mathcal{V}$ is reduced to $\{x\}$. Indeed, let $e_{1}$ and $e_{2}$ be two different elements of $\mathcal{E}$. Let $y \in \mathcal{V}$. Since $\eta \equiv \eta^{\prime}$ on $E \backslash E_{y}$, necessarily both $e_{1}$ and $e_{2}$ belong to $E_{y}$. We know also that both $e_{1}$ and $e_{2}$ belong to $E_{x}$. Consequently $e_{1} \in E_{x} \cap E_{y}$ and $e_{2} \in E_{x} \cap E_{y}$. It follows that $y=x$ since $e_{1}$ and $e_{2}$ are different. From (7.6), we deduce that, whenever $\mathcal{E}$ contains more than one element,

$$
\sum_{\sigma^{\prime} \in\{-1,1\}^{V}} c\left((\eta, \sigma),\left(\eta^{\prime}, \sigma^{\prime}\right)\right)=c\left((\eta, \sigma),\left(\eta^{\prime}, \sigma^{x}\right)\right) .
$$

Definition 7.1 and the three cases of equations (7.5), (7.7) and (7.8) complete the proof of Proposition 7.4.

From propositions 7.2, 7.3 and 7.4, we deduce the following theorem.

Theorem 7.5. Let $\left(\eta_{t}, \sigma_{t}\right)_{t \geq 0}$ be a Markov jump process with the transition rates introduced in definition $\%$.1. Then $\left(\eta_{t}, \sigma_{t}\right)_{t \geq 0}$ is reversible with respect to the coupling measure IP. Its second marginal process $\left(\sigma_{t}\right)_{t \geq 0}$ is a Markov process evolving according to a Glauber dynamics while its first marginal $\left(\eta_{t}\right)_{t \geq 0}$ is a non-Markovian jump process. 
Proof: The reversibility property is deduced from Proposition 7.2. Proposition 7.3 shows that the sum

$$
\sum_{\eta^{\prime} \in\{0,1\}^{E}} c\left((\eta, \sigma),\left(\eta^{\prime}, \sigma^{\prime}\right)\right)
$$

does not depend on $\eta$ for any $\sigma, \sigma^{\prime} \in\{-1,1\}^{V}$, moreover this sum is equal to the transition rates given in (5.5). The presence of the indicator function $\mathbb{I}_{\left(\eta^{\prime}, \sigma^{x}\right) \in \mathcal{C}_{x}}$ in the formula of proposition 7.4 shows that the sum $\sum_{\sigma^{\prime} \in\{-1,1\}^{V}} c\left((\eta, \sigma),\left(\eta^{\prime}, \sigma^{\prime}\right)\right)$ depends on $\sigma$ for some $\eta, \eta^{\prime} \in\{0,1\}^{E}$. The proof of Theorem 7.5 is complete thanks to Ball and Yeo (1993).

So this second dynamics does not fulfill our dream.

\section{The dream is not feasible}

In this section, we will work with the following additional hypothesis on the graph $(V, E)$.

Hypothesis 8.1. We suppose that there exists a vertex $\bar{x} \in V$ which belongs to at least four edges. We suppose that there exists a spin configuration $\bar{\sigma}$ such that $\delta_{\bar{\sigma}}(e)=0$ for any $e \in E$.

Recall that a graph is bipartite if its set of vertices can be partitioned into two parts in such a way that every edge connects a vertex in one part to a vertex in the other part. When the graph is bipartite, the spin configuration $\bar{\sigma}$ can be obtained by setting minuses on one part of the graph and pluses on the other part.

A Markov process on $\{0,1\}^{E} \times\{-1,1\}^{V}$ is said to be a Markovian coupling if both its marginals are Markov processes. Theorem 8.2 below shows that, under hypothesis 8.1, we cannot build a Markovian coupling which is reversible with respect to the $I P$ measure and whose marginal on the spins is a one spin flip Markov jump process, i.e., with transition rates satisfying (3.1).

Theorem 8.2. Suppose that hypothesis 8.1 holds. Let $\left(\eta_{t}, \sigma_{t}\right)_{t \geq 0}$ be a Markovian jump process with rates $q\left((\eta, \sigma),\left(\eta^{\prime}, \sigma^{\prime}\right)\right)$ satisfying (5.1), (5.2) and the detailed balance equation (5.3). If the second marginal $\left(\sigma_{t}\right)_{t \geq 0}$ is a Markov jump process with transition rates satisfying (3.1), then the first marginal $\left(\eta_{t}\right)_{t \geq 0}$ is a continuoustime non-Markovian jump process.

Proof: Let $\left(\eta_{t}, \sigma_{t}\right)_{t \geq 0}$ be a Markovian jump process with transition rates $q\left((\eta, \sigma),\left(\eta^{\prime}, \sigma^{\prime}\right)\right)$ satisfying (5.1), (5.2) and the detailed balance equation (5.3). Suppose also that the first marginal $\left(\eta_{t}\right)_{t \geq 0}$ is a Markov jump process with transition rates $\tilde{c}\left(\eta, \eta^{\prime}\right)$. Suppose also that the second marginal $\left(\sigma_{t}\right)_{t \geq 0}$ is a Markov jump process with transition rates $c\left(\sigma, \sigma^{\prime}\right)$ satisfying (3.1). From Ball and Yeo (1993) (see theorem A.2 of the appendix), we have

$$
\begin{gathered}
\forall \eta, \eta^{\prime} \in\{0,1\}^{E} \quad \forall \sigma \in\{-1,1\}^{V} \quad \sum_{\sigma^{\prime} \in\{-1,1\}^{V}} q\left((\eta, \sigma),\left(\eta^{\prime}, \sigma^{\prime}\right)\right)=\tilde{c}\left(\eta, \eta^{\prime}\right), \\
\forall \sigma, \sigma^{\prime} \in\{-1,1\}^{V} \quad \forall \eta \in\{0,1\}^{E} \quad \sum_{\eta^{\prime} \in\{0,1\}^{E}} q\left((\eta, \sigma),\left(\eta^{\prime}, \sigma^{\prime}\right)\right)=c\left(\sigma, \sigma^{\prime}\right) .
\end{gathered}
$$

Let $\bar{\sigma}$ be a configuration as in hypothesis 8.1 and let $\bar{x}$ be a vertex of $E$ belonging to at least four distinct edges. Let $\bar{\eta}$ be the configuration where all the edges are 
closed and let $\bar{\eta}^{\bar{x}}$ be the configuration where the edges exiting from $\bar{x}$ are opened, while all the other edges are closed. From formula (8.2) applied to $\bar{\eta}, \bar{\sigma}$ and $\bar{\sigma} \bar{x}$, we have

$$
\sum_{\eta^{\prime} \in\{0,1\}^{E}} q\left(\left(\bar{\eta}^{\bar{x}}, \bar{\sigma}^{\bar{x}}\right),\left(\eta^{\prime}, \bar{\sigma}\right)\right)=c\left(\bar{\sigma}^{\bar{x}}, \bar{\sigma}\right) .
$$

The only configuration $\eta^{\prime}$ compatible with $\bar{\sigma}$ is $\bar{\eta}$, thus the above identity reduces to

$$
q\left(\left(\bar{\eta}^{\bar{x}}, \bar{\sigma}^{\bar{x}}\right),(\bar{\eta}, \bar{\sigma})\right)=c\left(\bar{\sigma}^{\bar{x}}, \bar{\sigma}\right) .
$$

The conditions (3.1) implies that $c\left(\bar{\sigma}^{\bar{x}}, \bar{\sigma}\right)$ is positive, therefore $q\left(\left(\bar{\eta}^{\bar{x}}, \bar{\sigma}^{\bar{x}}\right),(\bar{\eta}, \bar{\sigma})\right)$ is positive. Since we assumed that the dynamics is reversible, then the rate $q\left((\bar{\eta}, \bar{\sigma}),\left(\bar{\eta}^{\bar{x}}, \bar{\sigma}^{\bar{x}}\right)\right)$ is also positive. From formula (8.1) applied to $\bar{\eta}, \bar{\eta}^{\bar{x}}$ and $\bar{\sigma}$, we have

$$
\sum_{\sigma^{\prime} \in\{-1,1\}^{V}} q\left((\bar{\eta}, \bar{\sigma}),\left(\bar{\eta}^{\bar{x}}, \sigma^{\prime}\right)\right)=\tilde{c}\left(\bar{\eta}, \bar{\eta}^{\bar{x}}\right),
$$

therefore the rate $\tilde{c}\left(\bar{\eta}, \bar{\eta}^{\bar{x}}\right)$ is positive. Let now $\widehat{\sigma}$ be a spin configuration in which the spins of the vertices connected to $\bar{x}$ contain at least two negative spins and at least two positive spins. This is possible because we assumed that $\bar{x}$ is connected to at least four distinct vertices. Applying again formula (8.1), this time to $\bar{\eta}, \bar{\eta}^{\bar{x}}$ and $\widehat{\sigma}$, we have

$$
\sum_{\sigma^{\prime} \in\{-1,1\}^{V}} q\left((\bar{\eta}, \widehat{\sigma}),\left(\bar{\eta}^{\bar{x}}, \sigma^{\prime}\right)\right)=\tilde{c}\left(\bar{\eta}, \bar{\eta}^{\bar{x}}\right) .
$$

Since the rate $\tilde{c}\left(\bar{\eta}, \bar{\eta}^{\bar{x}}\right)$ is positive, then there exists a spin configuration $\sigma^{\prime}$ such that

$$
q\left((\bar{\eta}, \widehat{\sigma}),\left(\bar{\eta}^{\bar{x}}, \sigma^{\prime}\right)\right)>0 .
$$

In the configuration $\bar{\eta}^{\bar{x}}$, all the edges emanating from $\bar{x}$ are opened, hence the neighbours of $\bar{x}$ are connected. Since the configuration $\sigma^{\prime}$ has to be compatible with $\bar{\eta}^{\bar{x}}$, then all the neighbours of $\bar{x}$ have the same spin in $\sigma^{\prime}$, hence the configuration $\sigma^{\prime}$ has to differ from $\widehat{\sigma}$ in at least two vertices. From the conditions (3.1), we should therefore have that $c\left(\widehat{\sigma}, \sigma^{\prime}\right)=0$. Yet formula (8.2) applied to $\bar{\eta}, \bar{\sigma}$ and $\bar{\sigma}^{\bar{x}}$ yields

$$
c\left(\widehat{\sigma}, \sigma^{\prime}\right)=\sum_{\eta^{\prime} \in\{0,1\}^{E}} q\left((\bar{\eta}, \widehat{\sigma}),\left(\eta^{\prime}, \sigma^{\prime}\right)\right) \geq q\left((\bar{\eta}, \widehat{\sigma}),\left(\bar{\eta}^{\bar{x}}, \sigma^{\prime}\right)\right)>0,
$$

which is contradictory.

Notice that hypothesis 8.1 holds for a box on the $d$-dimensional lattice when $d \geq 2$. Thus our dream process is not realizable in the lattice $\mathbb{Z}^{d}$ for $d \geq 2$.

\section{One edge and one incident cluster}

We drop here the requirement that the dynamics is local. We build a dynamics which updates at each time at most one edge and the vertices belonging to the open cluster of one of the endpoints of the edge. This dynamics will be reversible with respect to the coupling measure $I P$ and its marginal on the edges is the FK dynamics described in section 4. Recall that, for an edge $e=\langle x, y\rangle \in E$ and a configuration $\eta \in\{0,1\}^{E}, \gamma_{\eta}(e)=1$ if the endpoints $x, y$ of $e$ are connected by a path of open edges in $\eta$ which does not use the edge $e$ itself, and $\gamma_{\eta}(e)=0$ otherwise. The following definition gives the transition rates of the dynamics. 
Definition 9.1. Let $(\eta, \sigma),\left(\eta^{\prime}, \sigma^{\prime}\right)$ be two elements of $\{0,1\}^{E} \times\{-1,1\}^{V}$. We consider several cases:

- If $\sigma^{\prime}=\sigma$ and there exists $e \in E$ such that $\eta^{\prime}=\eta^{e}$ and $\gamma_{\eta}(e)=1$, then we set

$$
c\left((\eta, \sigma),\left(\eta^{\prime}, \sigma^{\prime}\right)\right)=\left((1-p) \mathbb{I}_{\eta(e)=1}+p \mathbb{I}_{\eta(e)=0}\right) \mathbb{I}_{(\eta, \sigma) \in \mathcal{C}} .
$$

- If $\sigma^{\prime}=\sigma$ and there exists $e \in E$ such that $\eta^{\prime}=\eta^{e}, \gamma_{\eta}(e)=0$ and $\delta_{\sigma}(e)=1$, then we set

$$
c\left((\eta, \sigma),\left(\eta^{\prime}, \sigma^{\prime}\right)\right)=\frac{1}{2}\left((1-p) \mathbb{I}_{\eta(e)=1}+p \mathbb{I}_{\eta(e)=0}\right) \mathbb{I}_{(\eta, \sigma) \in \mathcal{C}} .
$$

- If there exists $x \in V$ and $e \in E_{x}$ such that $\eta^{\prime}=\eta^{e}, \gamma_{\eta}(e)=0, \eta(e)=\delta_{\sigma}(e)$ and $\sigma^{\prime}$ is given by

$$
\forall y \in V \quad \sigma^{\prime}(y)= \begin{cases}\sigma(y) & \text { if } y \text { is not connected to } x \text { in } \eta \backslash\{e\}, \\ -\sigma(y) & \text { if } y \text { is connected to } x \text { in } \eta \backslash\{e\}\end{cases}
$$

then we set

$$
c\left((\eta, \sigma),\left(\eta^{\prime}, \sigma^{\prime}\right)\right)=\frac{1}{4}\left((1-p) \mathbb{1}_{\eta(e)=1} \mathbb{I}_{(\eta, \sigma) \in \mathcal{C}}+p \mathbb{\mathbb { I }}_{\eta(e)=0} \mathbb{\mathbb { I }}_{\left(\eta^{\prime}, \sigma^{\prime}\right) \in \mathcal{C}}\right) .
$$

- Otherwise, if $(\eta, \sigma) \neq\left(\eta^{\prime}, \sigma^{\prime}\right)$, then we set $c\left((\eta, \sigma),\left(\eta^{\prime}, \sigma^{\prime}\right)\right)=0$.

- Finally, if $(\eta, \sigma)=\left(\eta^{\prime}, \sigma^{\prime}\right)$, then we set

$$
c((\eta, \sigma),(\eta, \sigma))=-\sum_{\left(\eta^{\prime}, \sigma^{\prime}\right),\left(\eta^{\prime}, \sigma^{\prime}\right) \neq(\eta, \sigma)} c\left((\eta, \sigma),\left(\eta^{\prime}, \sigma^{\prime}\right)\right) .
$$

We first check that the dynamics associated to these rates is reversible with respect to the measure $I P$.

Proposition 9.2. The transition rates introduced in definition 9.1 satisfy the detailed balance equation (5.3).

Proof: Let $(\eta, \sigma)$ and $\left(\eta^{\prime}, \sigma^{\prime}\right)$ be fixed in $\{0,1\}^{E} \times\{-1,1\}^{V}$. We have to prove (5.3). We consider several cases, as in definition 9.1. Suppose first that $\sigma^{\prime}=\sigma$ and there exists $e \in E$ such that $\eta^{\prime}=\eta^{e}$ and $\gamma_{\eta}(e)=1$. We have then

$$
\begin{aligned}
& I P(\eta, \sigma) c\left((\eta, \sigma),\left(\eta^{\prime}, \sigma\right)\right)= \\
& \frac{1}{Z}\left(\prod_{f \in E}\left(p \mathbb{I}_{\eta(f)=1} \delta_{\sigma}(f)+(1-p) \mathbb{I}_{\eta(f)=0}\right)\right)\left((1-p) \mathbb{I}_{\eta(e)=1}+p \mathbb{I}_{\eta(e)=0}\right) \mathbb{I}_{(\eta, \sigma) \in \mathcal{C}} \\
& =\frac{1}{Z}\left(\prod_{f \in E \backslash\{e\}}\left(p \mathbb{I}_{\eta(f)=1}+(1-p) \mathbb{I}_{\eta(f)=0}\right)\right)(1-p) p \mathbb{I}_{(\eta, \sigma) \in \mathcal{C}} .
\end{aligned}
$$

We can remove the symbol $\delta_{\sigma}(f)$ in the product, thanks to the presence of the last indicator function, which guarantees that $\eta$ and $\sigma$ are compatible. Since $\eta^{\prime}=\eta^{e}$ and $\gamma_{\eta}(e)=1$, then $(\eta, \sigma) \in \mathcal{C}$ if and only if $\left(\eta^{\prime}, \sigma\right) \in \mathcal{C}$. Also, the product in the last formula is the same for $\eta$ and $\eta^{\prime}$, thus we obtain the detailed balance equation (5.3). Suppose next that $\sigma^{\prime}=\sigma$ and there exists $e \in E$ such that $\eta^{\prime}=\eta^{e}, \gamma_{\eta}(e)=0$ 
and $\delta_{\sigma}(e)=1$. We have then

$$
\begin{aligned}
& I P(\eta, \sigma) c\left((\eta, \sigma),\left(\eta^{\prime}, \sigma\right)\right)= \\
& \frac{1}{Z}\left(\prod_{f \in E}\left(p \mathbb{I}_{\eta(f)=1} \delta_{\sigma}(f)+(1-p) \mathbb{I}_{\eta(f)=0}\right)\right) \frac{1}{2}\left((1-p) \mathbb{I}_{\eta(e)=1}+p \mathbb{I}_{\eta(e)=0}\right) \mathbb{I}_{(\eta, \sigma) \in \mathcal{C}} \\
& =\frac{1}{Z}\left(\prod_{f \in E \backslash\{e\}}\left(p \mathbb{1}_{\eta(f)=1}+(1-p) \mathbb{1}_{\eta(f)=0}\right)\right) \frac{1}{2}(1-p) p \mathbb{I}_{(\eta, \sigma) \in \mathcal{C}} .
\end{aligned}
$$

Since $\eta^{\prime}=\eta^{e}$ and $\delta_{\sigma}(e)=1$, then $(\eta, \sigma) \in \mathcal{C}$ if and only if $\left(\eta^{\prime}, \sigma\right) \in \mathcal{C}$. Also, the product in the last formula is the same for $\eta$ and $\eta^{\prime}$, thus we obtain the detailed balance equation (5.3). Suppose finally that there exists $x \in V$ and $e \in E_{x}$ such that $\eta^{\prime}=\eta^{e}, \gamma_{\eta}(e)=0, \eta(e)=\delta_{\sigma}(e)$ and $\sigma^{\prime}$ is obtained from $\sigma$ by reversing all the spins of the sites which are connected to $x$ by an open path in $\eta \backslash\{e\}$. We have then

$$
\begin{aligned}
& I P(\eta, \sigma) c((\eta, \sigma),\left.\left(\eta^{\prime}, \sigma^{\prime}\right)\right) \\
&=\frac{1}{4 Z}(\left(\prod_{f \in E}\left(p \mathbb{I}_{\eta(f)=1} \delta_{\sigma}(f)+(1-p) \mathbb{I}_{\eta(f)=0}\right)\right) \\
& \times\left((1-p) \mathbb{I}_{\eta(e)=1} \mathbb{I}_{(\eta, \sigma) \in \mathcal{C}}+p \mathbb{I}_{\eta(e)=0} \mathbb{I}_{\left(\eta^{\prime}, \sigma^{\prime}\right) \in \mathcal{C}}\right) \\
&=\frac{1}{4 Z}\left(\prod_{f \in E \backslash\{e\}}\left(p \mathbb{I}_{\eta(f)=1}+(1-p) \mathbb{I}_{\eta(f)=0}\right)\right) \\
& \times(1-p) p\left(\mathbb{I}_{\eta(e)=1} \mathbb{I}_{(\eta, \sigma) \in \mathcal{C}}+\mathbb{I}_{\eta(e)=0} \mathbb{I}_{\left(\eta^{\prime}, \sigma^{\prime}\right) \in \mathcal{C}}\right) .
\end{aligned}
$$

To remove the symbol $\delta_{\sigma}(f)$ in the last line, we have used the fact that, if $\eta(e)=0$ and $\left(\eta^{\prime}, \sigma^{\prime}\right) \in \mathcal{C}$, then $(\eta, \sigma) \in \mathcal{C}$. Since we have

$$
\mathbb{I}_{\eta(e)=1} \mathbb{I}_{(\eta, \sigma) \in \mathcal{C}}+\mathbb{I}_{\eta(e)=0} \mathbb{I}_{\left(\eta^{\prime}, \sigma^{\prime}\right) \in \mathcal{C}}=\mathbb{I}_{\eta^{\prime}(e)=1} \mathbb{I}_{\left(\eta^{\prime}, \sigma^{\prime}\right) \in \mathcal{C}}+\mathbb{I}_{\eta^{\prime}(e)=0} \mathbb{I}_{(\eta, \sigma) \in \mathcal{C}},
$$

then we can conclude that

$$
I P(\eta, \sigma) c\left((\eta, \sigma),\left(\eta^{\prime}, \sigma^{\prime}\right)\right)=I P\left(\eta^{\prime}, \sigma^{\prime}\right) c\left(\left(\eta^{\prime}, \sigma^{\prime}\right),(\eta, \sigma)\right)
$$

and the detailed balance equation holds also in this case. We conclude that the detailed balance equation (5.3) is always satisfied.

The coefficients $\frac{1}{2}, \frac{1}{4}$ appearing in definition 9.1 have been chosen to ensure that the marginal dynamics on the edges is the FK dynamics presented in section 4. These coefficients are not important to ensure that the dynamics is reversible with respect to the measure $I P$. We compute next the marginal dynamics on the edges.

Proposition 9.3. Let $\eta$ be an element of $\{0,1\}^{E}$ and let $e \in E$. Let also $\sigma$ be an element of $\{-1,1\}^{V}$ such that $(\eta, \sigma) \in \mathcal{C}$.

- If $\gamma_{\eta}(e)=1$, then

$$
\sum_{\sigma^{\prime} \in\{-1,1\}^{V}} c\left((\eta, \sigma),\left(\eta^{e}, \sigma^{\prime}\right)\right)=p \mathbb{1}_{\eta(e)=0}+(1-p) \mathbb{I}_{\eta(e)=1} .
$$


- If $\gamma_{\eta}(e)=0$ and $\eta(e)=0$, then

$$
\sum_{\sigma^{\prime} \in\{-1,1\}^{V}} c\left((\eta, \sigma),\left(\eta^{e}, \sigma^{\prime}\right)\right)=\frac{p}{2} .
$$

- If $\gamma_{\eta}(e)=0$ and $\eta(e)=1$, then

$$
\sum_{\sigma^{\prime} \in\{-1,1\}^{V}} c\left((\eta, \sigma),\left(\eta^{e}, \sigma^{\prime}\right)\right)=1-p
$$

Proof: Let $\eta, e, \sigma$ be as in the statement of the proposition. We use the expression for the rates given in the definition 9.1. If $\gamma_{\eta}(e)=1$, then $c\left((\eta, \sigma),\left(\eta^{e}, \sigma^{\prime}\right)\right)$ is null unless $\sigma^{\prime}=\sigma$. Thus the sum reduces to $c\left((\eta, \sigma),\left(\eta^{e}, \sigma\right)\right)$, which is equal to $p$ if $\eta(e)=0$ and to $1-p$ if $\eta(e)=1$. Suppose next that $\gamma_{\eta}(e)=0$ and $\eta(e)=$ 0 . We consider two further cases. If $\delta_{\sigma}(e)=1$, then the sum reduces again to $c\left((\eta, \sigma),\left(\eta^{e}, \sigma\right)\right)$, which in this case is equal to $p / 2$. If $\delta_{\sigma}(e)=0$, then the sum contains two terms, corresponding to the two spin configurations $\sigma^{\prime}$ obtained from $\sigma$ by reversing all the spins of the sites which are connected to one extremity of $e$ by an open path in $\eta \backslash\{e\}$. These two rates $c\left((\eta, \sigma),\left(\eta^{e}, \sigma^{\prime}\right)\right)$ are both equal to $p / 4$, and their sum is again equal to $p / 2$. Suppose finally that $\gamma_{\eta}(e)=0$ and $\eta(e)=1$. The sum contains then three terms, one corresponding to $\sigma^{\prime}=\sigma$ and two corresponding to the two spin configurations $\sigma^{\prime}$ obtained from $\sigma$ by reversing all the spins of the sites which are connected to one extremity of $e$ by an open path in $\eta \backslash\{e\}$. The rate $c\left((\eta, \sigma),\left(\eta^{e}, \sigma\right)\right)$ is equal to $(1-p) / 2$. The two other rates $c\left((\eta, \sigma),\left(\eta^{e}, \sigma^{\prime}\right)\right)$ are equal to $(1-p) / 4$. In total, the sum is equal to $1-p$.

The marginal dynamics on the spins is quite complicated, but it is not a Markov process. Let us consider an edge configuration $\eta$ and a spin configuration $\sigma$ such that there exist $x \in V$ and $e \in E_{x}$ such that $\gamma_{\eta}(e)=\eta(e)=\delta_{\sigma}(e)=0$. Let $\sigma^{\prime}$ be the spin configuration defined by

$$
\forall y \in V \quad \sigma^{\prime}(y)= \begin{cases}\sigma(y) & \text { if } y \text { is not connected to } x \text { in } \eta \backslash\{e\} \\ -\sigma(y) & \text { if } y \text { is connected to } x \text { in } \eta \backslash\{e\} .\end{cases}
$$

We have then

$$
\sum_{\eta^{\prime} \in\{0,1\}^{E}} c\left((\eta, \sigma),\left(\eta^{\prime}, \sigma^{\prime}\right)\right)=c\left((\eta, \sigma),\left(\eta^{e}, \sigma^{\prime}\right)\right)=\frac{p}{4} .
$$

However the configuration $\sigma^{\prime}$ depends on $\eta$. Indeed, if we change the status of some edges $f$ in $E_{x} \backslash\{e\}$, we will change the set of the vertices which are connected to $x$ in $\eta \backslash\{e\}$, and we will obtain an edge configuration $\bar{\eta}$ for which

$$
\sum_{\eta^{\prime} \in\{0,1\}^{E}} c\left((\bar{\eta}, \sigma),\left(\eta^{\prime}, \sigma^{\prime}\right)\right)=c\left((\bar{\eta}, \sigma),\left(\bar{\eta}^{e}, \sigma^{\prime}\right)\right)=0 .
$$

Thus the above sum depends on the configuration $\eta$ and the marginal process on the spin configurations is not a Markov process. This remark, together with propositions 9.2 and 9.3, yield the following theorem.

Theorem 9.4. Let $\left(\eta_{t}, \sigma_{t}\right)_{t \geq 0}$ be a Markov jump process on $\mathcal{C}$ with the transition rates introduced in definition 7.1. Then $\left(\eta_{t}, \sigma_{t}\right)_{t \geq 0}$ is reversible with respect to the coupling measure IP. Its first marginal $\left(\eta_{t}\right)_{t \geq 0}$ is a Markov process evolving according to the FK dynamics while its second marginal process $\left(\sigma_{t}\right)_{t \geq 0}$ is a nonMarkovian jump process. 
Proof: The reversibility property is deduced from Proposition 9.2. Proposition 9.3 shows that the sum $\sum_{\sigma^{\prime} \in\{-1,1\}^{V}} c\left((\eta, \sigma),\left(\eta^{\prime}, \sigma^{\prime}\right)\right)$ does not depend on $\sigma$ for any $\eta, \eta^{\prime} \in\{0,1\}^{E}$, moreover this sum is equal to the transition rates given in (4.3). The proof of Theorem 9.4 is complete thanks to the remark before the theorem and the result of Ball and Yeo (1993).

So this third dynamics does not fulfill our dream, although it is not even local.

\section{One edge and one spin}

A notable inconvenience of the previous dynamics is that it might reverse simultaneously all the spins associated to an open cluster of the edge configuration. We introduce here a slight modification of this dynamics to ensure that at most one spin is changed at a time. For $x \in V$, recall that $E_{x}$ is the set of the edges $e$ in $E$ having $x$ as endvertex, i.e.,

$$
E_{x}=\{e \in E, e=\langle x, y\rangle, y \in V\} .
$$

The following definition gives the transition rates of the dynamics.

Definition 10.1. Let $(\eta, \sigma),\left(\eta^{\prime}, \sigma^{\prime}\right)$ be two elements of $\{0,1\}^{E} \times\{-1,1\}^{V}$. We consider several cases:

- If $\sigma^{\prime}=\sigma$ and there exists $e \in E$ such that $\eta^{\prime}=\eta^{e}$ and $\gamma_{\eta}(e)=1$, then we set

$$
c\left((\eta, \sigma),\left(\eta^{\prime}, \sigma^{\prime}\right)\right)=\left((1-p) \mathbb{I}_{\eta(e)=1}+p \mathbb{I}_{\eta(e)=0}\right) \mathbb{1}_{(\eta, \sigma) \in \mathcal{C}} .
$$

- If $\sigma^{\prime}=\sigma$ and there exists $e \in E$ such that $\eta^{\prime}=\eta^{e}, \gamma_{\eta}(e)=0$ and $\delta_{\sigma}(e)=1$, then we set

$$
c\left((\eta, \sigma),\left(\eta^{\prime}, \sigma^{\prime}\right)\right)=\frac{1}{2}\left((1-p) \mathbb{1}_{\eta(e)=1}+p \mathbb{1}_{\eta(e)=0}\right) \mathbb{I}_{(\eta, \sigma) \in \mathcal{C}} .
$$

- If there exists $x \in V$ and $e \in E_{x}$ such that $\sigma^{\prime}=\sigma^{x}, \eta^{\prime}=\eta^{e}, \gamma_{\eta}(e)=0$, $\eta(e)=\delta_{\sigma}(e)$ and $\eta(f)=0$ for $f \in E_{x} \backslash\{e\}$, then we set

$$
c\left((\eta, \sigma),\left(\eta^{\prime}, \sigma^{\prime}\right)\right)=\frac{1}{4}\left((1-p) \mathbb{I}_{\eta(e)=1} \mathbb{I}_{(\eta, \sigma) \in \mathcal{C}}+p \mathbb{1}_{\eta(e)=0} \mathbb{I}_{\left(\eta^{\prime}, \sigma^{\prime}\right) \in \mathcal{C}}\right) .
$$

- Otherwise, if $(\eta, \sigma) \neq\left(\eta^{\prime}, \sigma^{\prime}\right)$, then we set $c\left((\eta, \sigma),\left(\eta^{\prime}, \sigma^{\prime}\right)\right)=0$.

- Finally, if $(\eta, \sigma)=\left(\eta^{\prime}, \sigma^{\prime}\right)$, then we set

$$
c((\eta, \sigma),(\eta, \sigma))=-\sum_{\left(\eta^{\prime}, \sigma^{\prime}\right),\left(\eta^{\prime}, \sigma^{\prime}\right) \neq(\eta, \sigma)} c\left((\eta, \sigma),\left(\eta^{\prime}, \sigma^{\prime}\right)\right) .
$$

The difference compared to the rates of definition 9.1 is that, in definition 10.1, we allow to reverse the spins of a cluster only when it is reduced to a single vertex. This dynamics possesses the same properties as the dynamics of the previous section, namely, it is reversible with respect to the coupling measure IP, its first marginal process on the edges is a Markov process while its second marginal process on the spins is a non-Markovian jump process. The marginal dynamics on the edges differs from the FK dynamics in the following way. An edge can be opened between two different clusters only if one of the two clusters is reduced to a single vertex. More precisely, the corresponding rates are the following. For $x \in V$ and $e \in E_{x}$, for any configuration $\eta$,

$$
c\left(\eta, \eta^{e}\right)= \begin{cases}1-p & \text { if } \eta(e)=1 \\ p & \text { if } \eta(e)=0 \text { and } \gamma_{\eta}(e)=1, \\ p / 2 & \text { if } \eta(f)=0 \text { for } f \in E_{x} .\end{cases}
$$


Funnily enough, this fourth dynamics can be seen as a contraction of the first dynamics, which could make only one change at a time. The following proposition shows that, if the initial condition $\left(\eta_{0}, \sigma_{0}\right)$ is distributed as $I P$, then the infinitesimal behavior of the marginal on the spins of the process $\left(\eta_{t}, \sigma_{t}\right)_{t \geq 0}$, whose rates are introduced in definition 10.1, is quite similar to the transition rates of the Glauber dynamics as given in (5.5). The difference lies in the factor $\left|E_{x}\right|$. On the lattice $\mathbb{Z}^{d}$, the factor $\left|E_{x}\right|$ is equal to $2 d$ and is hence independent of $x$.

Proposition 10.2. Let $\left(\eta_{t}, \sigma_{t}\right)_{t \geq 0}$ be a Markov jump process with the transition rates introduced in definition 10.1. Suppose that the pair $\left(\eta_{0}, \sigma_{0}\right)$ is distributed according to $I P$. Then, for any $x \in V$, for any $\sigma \in\{-1,1\}^{V}$ and any $s \geq 0$,

$$
\lim _{t \rightarrow 0} \frac{1}{t} \mathbb{P}\left(\sigma_{t+s}=\sigma^{x} \mid \sigma_{s}=\sigma\right)=\frac{1}{4}\left|E_{x}\right| p(1-p)^{\left|\left\{e \in E_{x}: \delta_{\sigma}(e)=1\right\}\right|} .
$$

Proof: We have, for any $\sigma \in\{-1,1\}^{V}, x \in V$ and $s \geq 0$,

$$
\begin{aligned}
& \mathbb{P}\left(\sigma_{t+s}=\sigma^{x} \mid \sigma_{s}=\sigma\right) \\
& =\sum_{\eta \in\{0,1\}^{E}} \sum_{\eta^{\prime} \in\{0,1\}^{E}} \mathbb{P}\left(\sigma_{t+s}=\sigma^{x}, \eta_{t+s}=\eta^{\prime}, \eta_{s}=\eta \mid \sigma_{s}=\sigma\right) \\
& =\sum_{\eta \in\{0,1\}^{E}} \sum_{\eta^{\prime} \in\{0,1\}^{E}} \mathbb{P}\left(\sigma_{t+s}=\sigma^{x}, \eta_{t+s}=\eta^{\prime} \mid \sigma_{s}=\sigma, \eta_{s}=\eta\right) \mathbb{P}\left(\eta_{s}=\eta \mid \sigma_{s}=\sigma\right) .
\end{aligned}
$$

Recall that $\left(\eta_{t}, \sigma_{t}\right)_{t \geq 0}$ is a Markov jump process with transition rates given by $c\left((\eta, \sigma),\left(\eta^{\prime}, \sigma^{\prime}\right)\right)$, thus

$$
\lim _{t \rightarrow 0} \frac{1}{t} \mathbb{P}\left(\sigma_{t+s}=\sigma^{x}, \eta_{t+s}=\eta^{\prime} \mid \sigma_{s}=\sigma, \eta_{s}=\eta\right)=c\left((\eta, \sigma),\left(\eta^{\prime}, \sigma^{x}\right)\right)
$$

Using the expression of the rates $c\left((\eta, \sigma),\left(\eta^{\prime}, \sigma^{\prime}\right)\right)$ given in definition 10.1, together with formulas (10.2) and (10.3), we obtain that, for any $s \geq 0$,

$$
\begin{aligned}
\lim _{t \rightarrow 0} & \frac{1}{t} \mathbb{P}\left(\sigma_{t+s}=\sigma^{x} \mid \sigma_{s}=\sigma\right)=\sum_{\eta \in\{0,1\}^{E}} \sum_{e \in E_{x}}\left(\prod_{f \in E_{x} \backslash\{e\}} \mathbb{1}_{\eta(f)=0}\right) \\
& \times \frac{1}{4}\left((1-p) \mathbb{1}_{\eta(e)=1} \mathbb{I}_{(\eta, \sigma) \in \mathcal{C}}+p \mathbb{1}_{\eta(e)=0} \mathbb{I}_{\left(\eta^{e}, \sigma^{x}\right) \in \mathcal{C}}\right) \mathbb{P}\left(\eta_{s}=\eta \mid \sigma_{s}=\sigma\right) .
\end{aligned}
$$

The measure $I P$ is a reversible and a stationary measure for the process $\left(\eta_{t}, \sigma_{t}\right)_{t \geq 0}$. Hence, if $\left(\eta_{0}, \sigma_{0}\right)$ is distributed as $I P$, then, for any $s \geq 0,\left(\eta_{s}, \sigma_{s}\right)$ is also distributed as $I P$. Consequently, under the hypothesis of proposition 10.2 , we have

$$
\mathbb{P}\left(\eta_{s}=\eta \mid \sigma_{s}=\sigma\right)=\frac{\mathbb{P}\left(\eta_{s}=\eta, \sigma_{s}=\sigma\right)}{\mathbb{P}\left(\sigma_{s}=\sigma\right)}=\frac{I P(\eta, \sigma)}{\sum_{\eta^{\prime} \in\{0,1\}^{E}} I P\left(\eta^{\prime}, \sigma\right)}
$$

Equation (2.3) yields that

$$
\sum_{\eta^{\prime} \in\{0,1\}^{E}} I P\left(\eta^{\prime}, \sigma\right)=\frac{1}{Z}(1-p)^{\left|\left\{f \in E: \delta_{\sigma}(f)=0\right\}\right|} .
$$


Reporting (10.5) and (10.6) in (10.4), we conclude that

$$
\begin{aligned}
& \lim _{t \rightarrow 0} \frac{1}{t} \mathbb{P}\left(\sigma_{t+s}=\sigma^{x} \mid \sigma_{s}=\sigma\right)=\frac{1}{4} Z(1-p)^{-\left|\left\{f \in E: \delta_{\sigma}(f)=0\right\}\right|_{\times}} \\
& \sum_{\eta \in\{0,1\}^{E}} \sum_{e \in E_{x}}\left(\prod_{f \in E_{x} \backslash\{e\}} \mathbb{I}_{\eta(f)=0}\right)\left((1-p) \mathbb{I}_{\eta(e)=1} \mathbb{I}_{(\eta, \sigma) \in \mathcal{C}}+p \mathbb{I}_{\eta(e)=0} \mathbb{I}_{\left(\eta^{e}, \sigma^{x}\right) \in \mathcal{C}}\right) I P(\eta, \sigma) .
\end{aligned}
$$

We compute these sums as follows. We have, for $e \in E_{x}$,

$$
\begin{gathered}
\sum_{\eta \in\{0,1\}^{E}}\left(\prod_{f \in E_{x} \backslash\{e\}} \mathbb{1}_{\eta(f)=0}\right) \mathbb{1}_{\eta(e)=1} \mathbb{I}_{(\eta, \sigma) \in \mathcal{C}} I P(\eta, \sigma)= \\
\frac{1}{Z}(1-p)^{\left|E_{x}\right|-1+\left|\left\{f \in E \backslash E_{x}: \delta_{\sigma}(f)=0\right\}\right|} p \delta_{\sigma}(e), \\
\sum_{\eta \in\{0,1\}^{E}}\left(\prod_{f \in E_{x} \backslash\{e\}} \mathbb{1}_{\eta(f)=0}\right) \mathbb{1}_{\eta(e)=0} \mathbb{I}_{\left(\eta^{e}, \sigma^{x}\right) \in \mathcal{C}} I P(\eta, \sigma)= \\
\frac{1}{Z}(1-p)^{\left|E_{x}\right|+\left|\left\{f \in E \backslash E_{x}: \delta_{\sigma}(f)=0\right\}\right|}\left(1-\delta_{\sigma}(e)\right) .
\end{gathered}
$$

Reporting the values of these sums in formula (10.7), we obtain

$$
\begin{aligned}
& \lim _{t \rightarrow 0} \frac{1}{t} \mathbb{P}\left(\sigma_{t+s}=\sigma^{x} \mid \sigma_{s}=\sigma\right)=\frac{1}{4}(1-p)^{-\left|\left\{f \in E: \delta_{\sigma}(f)=0\right\}\right|} \times \\
& \left.\sum_{e \in E_{x}}(1-p)^{\left|E_{x}\right|+\left|\left\{f \in E \backslash E_{x}: \delta_{\sigma}(f)=0\right\}\right|} p \delta_{\sigma}(e)+p(1-p)^{\left|E_{x}\right|+\left|\left\{f \in E \backslash E_{x}: \delta_{\sigma}(f)=0\right\}\right|}\left(1-\delta_{\sigma}(e)\right)\right) \\
& \quad=\frac{1}{4}(1-p)^{-\left|\left\{f \in E: \delta_{\sigma}(f)=0\right\}\right|} \sum_{e \in E_{x}} p(1-p)^{\left|E_{x}\right|+\left|\left\{f \in E \backslash E_{x}: \delta_{\sigma}(f)=0\right\}\right|} \\
& =\frac{1}{4} \sum_{e \in E_{x}} p(1-p)^{\left|\left\{f \in E_{x}: \delta_{\sigma}(f)=1\right\}\right|}=\frac{1}{4}\left|E_{x}\right| p(1-p)^{\left|\left\{f \in E_{x}: \delta_{\sigma}(f)=1\right\}\right|}
\end{aligned}
$$

This yields the desired result (10.1).

\section{Appendix A. Appendix}

Let $(V, E)$ be a finite graph and let $\left(X_{t}\right)_{t \geq 0}=\left(\left(\eta_{t}, \sigma_{t}\right)\right)_{t \geq 0}$ be a time-homogeneous continuous-time Markov process with state space $\Omega=\{0,1\}^{E} \times\{-1,1\}^{V}$ and with infinitesimal generator $Q$ defined as follows. For any function $f$ defined on $\Omega$ with values in $\mathbb{R}$, we have

$$
Q f(\eta, \sigma)=\sum_{\left(\eta^{\prime}, \sigma^{\prime}\right) \in \Omega} q\left((\eta, \sigma),\left(\eta^{\prime}, \sigma^{\prime}\right)\right)\left(f\left(\eta^{\prime}, \sigma^{\prime}\right)-f(\eta, \sigma)\right),
$$

where $q\left((\eta, \sigma),\left(\eta^{\prime}, \sigma^{\prime}\right)\right)$ are the transition rates defined by, for $(\eta, \sigma) \neq\left(\eta^{\prime}, \sigma^{\prime}\right)$,

$$
q\left((\eta, \sigma),\left(\eta^{\prime}, \sigma^{\prime}\right)\right)=\lim _{t \rightarrow 0^{+}} \frac{1}{t} \mathbb{P}\left(X_{t}=\left(\eta^{\prime}, \sigma^{\prime}\right) \mid X_{0}=(\eta, \sigma)\right),
$$

and for $(\eta, \sigma)=\left(\eta^{\prime}, \sigma^{\prime}\right)$,

$$
q((\eta, \sigma),(\eta, \sigma))=-\sum_{\left(\eta^{\prime}, \sigma^{\prime}\right),(\eta, \sigma) \neq\left(\eta^{\prime}, \sigma^{\prime}\right)} q\left((\eta, \sigma),\left(\eta^{\prime}, \sigma^{\prime}\right)\right) .
$$


Our goal is to present some conditions on the transition rates $q\left((\eta, \sigma),\left(\eta^{\prime}, \sigma^{\prime}\right)\right)$ under which $\left(\sigma_{t}\right)_{t \geq 0}$ or $\left(\eta_{t}\right)_{t \geq 0}$ are Markov processes. This is a lumpability problem discussed in Ball and Yeo (1993). We first recall the following definition of lumpability.

Definition A.1. Let $\Omega$ be a countable set, let $\Omega_{1}, \cdots, \Omega_{r}$ be a partition of $\Omega$ and let $h$ be the function from $\Omega$ to $\{1, \cdots, r\}$ defined by $h(x)=j$ if $x \in \Omega_{j}$. An homogeneous Markov chain $\left(X_{t}\right)_{t \geq 0}$ with state space $\Omega$ is lumpable with respect to the partition $\Omega_{1}, \cdots, \Omega_{r}$ if $\left(h\left(X_{t}\right)\right)_{t \geq 0}$ is an homogeneous Markov chain for every initial distribution $y_{0}$ on $\{1, \cdots, r\}$ and its transition rates do not depend on the choice of the initial distribution $y_{0}$.

We define

$$
\forall \sigma \in\{-1,1\}^{V} \quad \Omega_{\sigma}=\left\{(\eta, \sigma) \in \Omega: \eta \in\{0,1\}^{E}\right\}
$$

and we consider the corresponding partition of $\Omega$ :

$$
\Omega=\bigcup_{\sigma \in\{-1,1\}^{V}} \Omega_{\sigma} .
$$

In this case, the function $h$ is the projection from $\Omega$ to $\{-1,1\}^{V}$ defined by $h(\eta, \sigma)=\sigma$. Our purpose is to obtain necessary and sufficient conditions under which the Markov process $\left(\eta_{t}, \sigma_{t}\right)_{t \geq 0}$ is lumpable with respect to the partition $\left(\Omega_{\sigma}, \sigma \in\{-1,1\}^{V}\right)$. It is well known (see for instance ${ }^{1}$ Theorem 3.1 in Ball and Yeo, 1993) that a Markov process is lumpable with respect to the partition $\left(\Omega_{j}\right)_{1 \leq j \leq r}$ if and only if there exist positive real numbers $\lambda_{i, j}, 1 \leq i, j \leq r$, such that

$$
\forall i \neq j, \quad \forall x \in \Omega_{i}, \quad \sum_{y \in \Omega_{j}} q_{x, y}=\lambda_{i, j} .
$$

In order to simplify the reading of this paper, we adapt this criterion of lumpability to the case of the partition $\left(\Omega_{\sigma}, \sigma \in\{-1,1\}^{V}\right)$ and the Markov process $\left(\eta_{t}, \sigma_{t}\right)_{t \geq 0}$. We summarize it in the following theorem.

Theorem A.2. Let $\left(\eta_{t}, \sigma_{t}\right)_{t \geq 0}$ be a continuous-time Markov process with finite state space $\{0,1\}^{E} \times\{-1,1\}^{V}$ and transition rates $q\left((\eta, \sigma),\left(\eta^{\prime}, \sigma^{\prime}\right)\right)$. Then the following statements are equivalent:

(i) For any $\sigma \neq \sigma^{\prime}$, there exist positive numbers $c\left(\sigma, \sigma^{\prime}\right)$ such that

$$
\forall \eta \in\{0,1\}^{E}, \quad \sum_{\eta^{\prime} \in\{0,1\}^{E}} q\left((\eta, \sigma),\left(\eta^{\prime}, \sigma^{\prime}\right)\right)=c\left(\sigma, \sigma^{\prime}\right) .
$$

(ii) The spin marginal $\left(\sigma_{t}\right)_{t \geq 0}$ of the Markov process $\left(\left(\eta_{t}, \sigma_{t}\right)\right)_{t \geq 0}$ is a Markov process for every initial distribution $\sigma_{0}$ on $\{-1,1\}^{V}$ and its transition rates $c\left(\sigma, \sigma^{\prime}\right)$ do not depend on the choice of the initial distribution of $\sigma_{0}$.

The analog of Theorem A.2 holds for the edge marginal process of $\left(\eta_{t}, \sigma_{t}\right)_{t \geq 0}$. For a proof, we refer to Theorem 3.1 in Ball and Yeo (1993). We also recall Theorem 3.2 of Ball and Yeo (1993), that we adapt to our context.

\footnotetext{
${ }^{1}$ Ball and Yeo's result is stated under their condition 2.2 which is satisfied in the current context.
} 
Theorem A.3. Suppose that the transition rates $q\left((\eta, \sigma),\left(\eta^{\prime}, \sigma^{\prime}\right)\right)$ of the Markov process $\left(\eta_{t}, \sigma_{t}\right)_{t \geq 0}$ satisfy

$q\left((\eta, \sigma),\left(\eta^{\prime}, \sigma^{\prime}\right)\right) \neq 0 \Longrightarrow(\eta, \sigma)=\left(\eta^{\prime}, \sigma^{\prime}\right)$ or $\left(\eta=\eta^{\prime}, \quad \sigma \neq \sigma^{\prime}\right)$ or $\left(\eta \neq \eta^{\prime}, \quad \sigma=\sigma^{\prime}\right)$. Then $\left(\eta_{t}\right)_{t \geq 0}$ and $\left(\sigma_{t}\right)_{t \geq 0}$ are both Markov processes if and only if they are mutually independent.

\section{References}

F. Ball and G. F. Yeo. Lumpability and marginalisability for continuous-time Markov chains. J. Appl. Probab. 30 (3), 518-528 (1993). MR1232731.

R. G. Edwards and A. D. Sokal. Generalization of the Fortuin-Kasteleyn-SwendsenWang representation and Monte Carlo algorithm. Phys. Rev. D (3) 38 (6), 2009-2012 (1988). MR965465.

G. Grimmett. The stochastic random-cluster process and the uniqueness of randomcluster measures. Ann. Probab. 23 (4), 1461-1510 (1995). MR1379156.

G. Grimmett. The random-cluster model, volume 333 of Grundlehren der Mathematischen Wissenschaften [Fundamental Principles of Mathematical Sciences]. Springer-Verlag, Berlin (2006). ISBN 978-3-540-32890-2; 3-540-32890-4. MR2243761.

O. Häggström. Dynamical percolation: early results and open problems. In $M i$ crosurveys in discrete probability (Princeton, NJ, 1997), volume 41 of DIMACS Ser. Discrete Math. Theoret. Comput. Sci., pages 59-74. Amer. Math. Soc., Providence, RI (1998). MR1630409.

T. M. Liggett. Interacting particle systems. Classics in Mathematics. SpringerVerlag, Berlin (2005). ISBN 3-540-22617-6. MR2108619.

E. Olivieri and M. E. Vares. Large deviations and metastability, volume 100 of Encyclopedia of Mathematics and its Applications. Cambridge University Press, Cambridge (2005). ISBN 0-521-59163-5. MR2123364.

R. H. Schonmann. Slow droplet-driven relaxation of stochastic Ising models in the vicinity of the phase coexistence region. Comm. Math. Phys. 161 (1), 1-49 (1994). MR1266068.

J. E. Steif. A survey of dynamical percolation. In Fractal geometry and stochastics IV, volume 61 of Progr. Probab., pages 145-174. Birkhäuser Verlag, Basel (2009). MR2762676. 